\title{
Pueraria lobata and Daidzein Reduce Cytotoxicity by Enhancing Ubiquitin-Proteasome System Function in SCA3-iPSC- Derived Neurons
}

\author{
I-Cheng Chen $\mathbb{D}^{1},{ }^{1}$ Kuo-Hsuan Chang ${ }^{D},{ }^{1}$ Yi-Jing Chen, ${ }^{1}$ Yi-Chun Chen, ${ }^{1}$ \\ Guey-Jen Lee-Chen $\mathbb{D}^{2}$ and Chiung-Mei Chen $\mathbb{D}^{1}$
}

${ }^{1}$ Department of Neurology, Chang Gung Memorial Hospital, Chang Gung University College of Medicine, Taoyuan 33302, Taiwan

${ }^{2}$ Department of Life Science, National Taiwan Normal University, Taipei 11677, Taiwan

Correspondence should be addressed to Chiung-Mei Chen; cmchen@adm.cgmh.org.tw

I-Cheng Chen and Kuo-Hsuan Chang contributed equally to this work.

Received 20 February 2019; Revised 7 July 2019; Accepted 23 July 2019; Published 7 October 2019

Academic Editor: Aldrin V. Gomes

Copyright (C) 2019 I-Cheng Chen et al. This is an open access article distributed under the Creative Commons Attribution License, which permits unrestricted use, distribution, and reproduction in any medium, provided the original work is properly cited.

\begin{abstract}
Spinocerebellar ataxia type 3 (SCA3) is an autosomal dominant neurodegenerative disorder caused by a CAG repeat expansion within the ATXN3/MJD1 gene. The expanded CAG repeats encode a polyglutamine (polyQ) tract at the C-terminus of the ATXN3 protein. ATXN3 containing expanded polyQ forms aggregates, leading to subsequent cellular dysfunctions including an impaired ubiquitin-proteasome system (UPS). To investigate the pathogenesis of SCA3 and develop potential therapeutic strategies, we established induced pluripotent stem cell (iPSC) lines from SCA3 patients (SCA3-iPSC). Neurons derived from SCA3-iPSCs formed aggregates that are positive to the polyQ marker 1C2. Treatment with the proteasome inhibitor, MG132, on SCA3-iPSC-derived neurons downregulated proteasome activity, increased production of radical oxygen species (ROS), and upregulated the cleaved caspase 3 level and caspase 3 activity. This increased susceptibility to the proteasome inhibitor can be rescued by a Chinese herbal medicine (CHM) extract NH037 (from Pueraria lobata) and its constituent daidzein via upregulating proteasome activity and reducing protein ubiquitination, oxidative stress, cleaved caspase 3 level, and caspase 3 activity. Our results successfully recapitulate the key phenotypes of the neurons derived from SCA3 patients, as well as indicate the potential of NH037 and daidzein in the treatment for SCA3 patients.
\end{abstract}

\section{Introduction}

Spinocerebellar ataxia type 3 (SCA3, also known as MachadoJoseph disease (MJD)) is an autosomal dominant neurodegenerative disease and thought to be the most common subtype of SCA worldwide $[1,2]$. SCA3 is caused by an expanded CAG repeats within the $A T X N 3 / M J D 1$ gene on chromosome 14q32.1 [3]. The most common isoform of ATXN3 expressed in the brain contains 11 exons and is translated into approximately $42 \mathrm{kDa}$ ATXN3 protein depending on the length of CAG repeats encoding a polyglutamine (polyQ) tract at the C-terminus of the ATXN3 protein [4]. ATXN3 protein is a deubiquitinating enzyme that plays an important role in the ubiquitin-proteasome system (UPS) that regulates protein degradation [5]. The Josephin domain together with the ubiquitin-interacting motifs deubiquitinates proteins to regenerate reusable ubiquitin $[5,6]$. Besides the role in protein degradation, ATXN3 regulates transcription by interacting with numerous key regulators of transcription and also by directly binding to DNA through a leucine zipper motif [7].

The expanded polyQ tract ( $>51$ repeats) in ATXN3 causes conformational changes, resulting in formation of protein aggregates and altered ATXN3 function. In the brains of SCA3 patients, mutant ATXN3 protein tends to accumulate in neuronal cell nuclei [8]. However, cytosolic aggregates are also found in mutant ATXN3-overexpressing 
COS-7 cells, suggesting a widespread aggregation of polyQexpanded ATXN3 protein [9]. Aggregates formed by polyQexpanded ATXN3 result in subsequent cellular dysfunctions including transcriptional dysregulation, mitochondrial function, and misfolded protein clearance, eventually leading to cell death and neurodegeneration [10].

Up to now, there is no effective treatment available for polyQ disorders including SCA3. Activation of autophagy and UPS could be potential therapeutic targets for SCA3. Overexpression of BECLIN-1 improved the clearance of mutant ATXN3 in rats [11]. Treatment with rapamycin to upregulate autophagy in SCA3 mice could decrease mutant ATXN3 aggregation [12]. Recently, cordycepin, a bioactive compound in the fungi Cordyceps and a derivative of the nucleoside adenosine, was found to activate autophagy through AMPK phosphorylation to reduce behavioral and neuropathological phenotypes in SCA3 mice [13]. Given that impairment of UPS plays an important role in pathogenesis of polyQ diseases including SCA3 [14], activation of UPS has also been implicated as a probable therapeutic target for SCA3 $[15,16]$. Recently, lines of evidence have shown that traditional Chinese medicine is a potential strategy for the treatment of neurodegenerative diseases. For example, Ginkgo biloba extract EGb 761 has been reported to increase proteasome activity and enhance degradation of aggregated protein in a Huntington's disease (HD) cellular model [17]. Tianma (Rhizoma gastrodiae) modulates proteins related to the UPS in differentiated SH-SY5Y cells [18]. Previously, we also found that Pueraria lobata (NH037) and its active constituent daidzein significantly enhanced UPS function and proteasome activity, reduced ATXN3 polyQ aggregation, improved neurite outgrowth, and displayed antiapoptotic effects in ATXN3/Q 75 -GFP 293/SH-SY5Y cell models, whereas NH037 and daidzein did not activate autophagy in a HEK293 cell model [19]. Through promoting UPS to provide neuroprotection, NH037 and daidzein could be a potential candidate for developing a therapeutic strategy of SCA3.

Up to now, the available treatment to halt the neurodegeneration of SCA3 is still absent. The lack of live neurons from patients to investigate the molecular details of neurodegeneration may be a bottleneck for drug research in SCA3. Recent progress in the induced pluripotent stem cell (iPSC) technology offers a unique opportunity to acquire neurons carrying an identical genetic background of SCA3 patients to investigate the potential pathogenesis and therapeutic strategies for SCA3. In this study, we established iPSCs from SCA3 patients (SCA3-iPSCs), which were differentiated into neurons characterized by polyQ aggregates and impaired UPS. We further examined the neuroprotective potentials of NH037 and daidzein on the neurons derived from SCA3-iPSCs. Our findings provide a new disease-specific model for SCA3, which can also serve as a platform to identify new treatments for neurodegenerative diseases.

\section{Materials and Methods}

2.1. Derivation of Human Skin Fibroblasts. Briefly, human skin fibroblasts were obtained from two normal controls (NC-1: 63-year-old female; NC-2: 31-year-old male) and two SCA3 patients (SCA3-1: 47-year-old female, 66 repeats;
SCA3-2: 50-year-old male, 68 repeats). Isolation and cultivation of human skin fibroblasts were performed as described previously [20].

\subsection{Retroviral Reprogramming and Generation of Human} iPSC Lines. Human skin fibroblasts were reprogrammed by four factor combinations including OCT4, SOX2, KLF4, and c-MYC, and mouse embryonic fibroblast (MEF) feeder cells from 13.5 to $14.5 \mathrm{dpc}$ CD1 mouse embryos were prepared using a standard method as previously described [21]. The reprogrammed human iPSCs were maintained on mitomycin C-deactivated MEF feeder cells in Knockout Dulbecco's modified Eagle medium (DMEM, Invitrogen, Waltham, MA, USA) supplemented with $20 \%$ Knockout Serum Replacement (KSR, Invitrogen), 8 ng/ml bFGF (Peprotech, Rocky Hill, NJ, USA), $50 \mathrm{U} / \mathrm{ml}$ penicillin, $50 \mathrm{mg} / \mathrm{ml}$ streptomycin, $1 \mathrm{mM}$ sodium pyruvate, $1 \mathrm{x}$ MEM nonessential amino acid, $2 \mathrm{mM}$ L-glutamine, and 0.1 mM 2-mercaptoethanol.

2.3. SCA3 Genotyping. Genomic DNA from iPSCs and parental fibroblasts was extracted by a DNA extraction kit (Qiagen, Hilden, Germany) and amplified by PCR analysis with ATXN3 specific primers (forward: CCAGTGACTACTTTGATTCG, reverse: CTTACCTAGATCACTCCCAA). Annealing temperature was at $54^{\circ} \mathrm{C}$, and the length of PCR fragments ranges from $235 \mathrm{bp}$ to $403 \mathrm{bp}$ (corresponding to $12 \sim 68$ CAG repeats).

2.4. Immunocytochemistry. Briefly, cells were fixed in $4 \%$ paraformaldehyde for $20 \mathrm{~min}$, permeabilized with $0.05 \%$ Triton X-100 in PBS for $20 \mathrm{~min}$, and then blocked in 1\% BSA for at least 1 hour. Cells were then incubated with primary antibodies and corresponding secondary antibodies for further examination. For detecting polyQ aggregation, primary antibodies 1C2 (1:200, Millipore, Burlington, MA, USA) and P62 ( $1: 200$, Santa Cruz, Dallas, TX, USA) were used. Images were captured by a fluorescence microscope (DMI4000 B, Leica, Wetzlar, Germany) and processed by MetaMorph microscopy automation and image analysis software (Molecular Devices, Sunnyvale, CA, USA).

2.5. Characterization of Human iPSC Colonies. Alkaline phosphatase activity of the reprogrammed iPSC colonies was tested using an alkaline phosphatase detection kit (Millipore) according to the manufacturer's instructions. Immunocytochemistry was performed to confirm pluripotency marker expression (OCT4, 1:200, and TRA-1-81, 1:200, Abcam, Cambridge, UK). RT-PCR, karyotyping, and hematoxylin and eosin staining (HE staining) of teratoma tissues were performed as previously described [17].

2.6. Monolayer Neuronal Differentiation from iPSC Lines. iPSC cultures were disaggregated using Accutase (Millipore) and preplated on gelatin for 1 hour at $37^{\circ} \mathrm{C}$ to remove MEFs. The feeder-free iPSCs were plated on Matrigel (BD, Franklin Lakes, NJ, USA)-coated dishes in MEF-conditioned iPSC medium spiked with $8 \mathrm{ng} / \mathrm{ml}$ of bFGF. The initial differentiation medium conditions were N2B27 medium (1:1 mixture of DMEM/F12 supplemented with modified N2 and Neurobasal medium supplemented with B27, all purchased from Invitrogen) spiked with $10 \mu \mathrm{M}$ of the $\mathrm{TGF} \beta$ inhibitor 
SB431542 (Sigma-Aldrich, St. Louis, MO, USA). Neural progenitors were obtained and maintained with bFGF ( $10 \mathrm{ng} / \mathrm{ml})$ and EGF (10 ng/ml, Peprotech) in poly-L-lysinecoated plates then characterized by immunostaining with neural stem cell (NSC) markers NESTIN (1:200, DSHB, Iowa City, IA, USA) and PAX6 (1:200, Covance, Princeton, NJ, USA). After supplementation with $20 \mathrm{ng} / \mathrm{ml}$ BDNF (Peprotech), $0.2 \mathrm{mM}$ ascorbic acid (Sigma-Aldrich), $10 \mathrm{ng} / \mathrm{ml} \mathrm{GDNF}$ (Peprotech), and $1 \mathrm{ng} / \mathrm{ml}$ TGF $\beta 3$ (Peprotech) for two weeks, matured neurons were confirmed by TUJ1 (1:500, Covance).

2.7. Trypan Blue Exclusion Assay. For the cell viability assay, $20 \mu \mathrm{l}$ of $0.4 \%$ trypan blue (Invitrogen) was mixed with $20 \mu \mathrm{l}$ cell suspension from each sample and viable cells that excluded trypan blue were determined by light microscopy.

2.8. Caspase 3 Activity Measurement. Cells were lysed in $1 \mathrm{x}$ lysis buffer by repeated cycles of freezing and thawing. Caspase 3 activity was measured with the caspase 3 assay kit according to the manufacturer's instructions (Sigma-Aldrich).

2.9. Proteasome Activity Assay. Proteasome activity was determined using a proteasome activity assay kit (Abcam) with chymotrypsin-like (SUC-LLVY-AMC) substrates according to the manufacturer's protocol. Fluorescence was read using a 380/460 nm filter set in a fluorometer (FLx800 fluorescence microplate reader, BioTek, Winooski, VT, USA).

2.10. Filter Trap Assay. Cells were lysed in RIPA buffer, and protein samples were filtered through a cellulose acetate membrane $(0.2 \mu \mathrm{m}$ pore size $)$ by Bio-Dot ${ }^{\circledR}$ Microfiltration Apparatus (Bio-Rad, Hercules, CA, USA). The membrane was blocked in PBS containing 10\% nonfat dried milk overnight, and the mouse $1 \mathrm{C} 2$ antibody (1:500, Millipore) was used to detect aggregation.

2.11. Cellular Radical Oxygen Species (ROS) Analysis. To measure oxidative stress induced by MG132, a fluorogenic reagent (CellROX ${ }^{\mathrm{TM}}$ Deep Green, Molecular Probes, Eugene, OR, USA) was added to cells at a final concentration of $5 \mu \mathrm{M}$ and incubated at $37^{\circ} \mathrm{C}$ for $30 \mathrm{~min}$. Cells were washed with PBS and fixed in $4 \%$ formaldehyde. Images were captured by a fluorescence microscope (DMI4000 B, Leica) and processed by MetaMorph microscopy automation and image analysis software (Molecular Devices).

2.12. Lactate Dehydrogenase (LDH) Cell Toxicity Assay. Culture media were collected, and $100 \mu \mathrm{l}$ of supernatant from each sample was transferred to a 96-well plate to determine the release of $\mathrm{LDH}$ by the $\mathrm{LDH}$ cytotoxicity assay kit (Cayman, Ann Arbor, MI, USA). The absorbance was read at $490 \mathrm{~nm}$ with a microplate reader (Multiskan GO, Thermo Scientific, Waltham, MA, USA).

2.13. Western Blot Analysis. Cells were lysed in lysis buffer (1 mM EDTA, $1 \mathrm{mM}$ PMSF, $5 \mu \mathrm{g} / \mathrm{ml}$ aprotinin, $150 \mathrm{mM}$ $\mathrm{NaCl}, 50 \mathrm{mM}$ Tris- $\mathrm{HCl} \mathrm{pH}$ 7.4, $5 \mu \mathrm{g} / \mathrm{ml}$ leupeptin, $1 \%$ sodium deoxycholate, $1 \%$ Triton X-100, and $0.1 \%$ SDS). After sonication and sitting on ice for $20 \mathrm{~min}$, the lysates were centrifuged at $14000 \times \mathrm{g}$ for $10 \mathrm{~min}$ at $4^{\circ} \mathrm{C}$. Protein concen- trations were determined using the Bio-Rad protein assay kit (Bio-Rad), with albumin as standards. Total proteins $(20 \sim 30 \mu \mathrm{g})$ were electrophoresed on $10 \sim 12 \%$ SDSpolyacrylamide gel and transferred onto a nitrocellulose membrane (Bio-Rad) by reverse electrophoresis. After being blocked by $10 \%$ milk, the membrane is stained with caspase 3 (1:1000; Cell Signaling, Danvers, MA, USA), ubiquitin (1:500; Dako, Santa Clara, CA, USA), and TUJ1 (1:5000; Covance) primary antibodies. The immune complexes are detected using horseradish peroxidase-conjugated goat antimouse (Jackson ImmunoResearch, West Grove, PA, USA) or goat anti-rabbit (Rockland, Pottstown, PA, USA) IgG antibody (1:10000 dilution) and Immobilon ${ }^{\mathrm{TM}}$ Western Chemiluminescent HRP substrate (Millipore).

2.14. Lipid Peroxidation Malondialdehyde (MDA) Assay. Oxidative stress was evaluated by the MDA assay kit (Abcam). Cells were prepared and examined according to the manufacturer's protocol. The fluorescence was read at $532 \mathrm{~nm}$ in a microplate reader (Multiskan GO, Thermo Scientific).

2.15. Herbal Extract and Compounds/Chemicals. Chinese herbal extract $\mathrm{NH} 037(1 \mathrm{mg} / \mathrm{ml}$ as final working concentration) was provided by Sun Ten Pharmaceutical Co. (New Taipei City, Taiwan), and the preparation was performed as previously described [22]. Daidzein (50 $\mu \mathrm{M})$ and MG132 $(2 \mu \mathrm{M})$ were purchased from Sigma-Aldrich.

2.16. Statistical Analysis. For each set of values, data are represented as the mean \pm standard deviation $(\mathrm{SD})$ of three independent experiments. Differences between groups were evaluated by two-tailed Student's $t$-test or ANOVA (one-way and two-way) with a post hoc LSD test where appropriate. A $p$ value less than 0.05 was considered statistically significant.

\section{Results}

3.1. Generation and Neural Differentiation of iPSCs from SCA3 Patients. To establish iPSCs for SCA3, primary dermal fibroblasts from two SCA3 patients (SCA3-1: 47-year-old female; SCA3-2: 50-year-old male) and two normal controls (NC-1: 63-year-old female; NC-2: 31-year-old male) were transfected with four reprogramming factors OCT4, KLF4, SOX2, and c-MYC by retroviruses. Human ESC-like colonies were generated three to four weeks after transfection (Figure 1(a)). PCR analysis of genomic DNA showed that patients' iPSCs (SCA3-1 and SCA3-2) contain mutant ATXN3 CAG repeats (SCA3-1 with 66 repeats and SCA3-2 with 68 repeats, Figure 1(b)). These iPSCs were positive for pluripotent markers alkaline phosphatase, OCT4, and TRA1-81 (Figure 1(c)). RT-PCR analysis of iPSCs indicated that exogenous OCT4, KLF4, SOX2, and $c-M Y C$ genes delivered by retroviruses for reprogramming were silenced after passage. In contrast, endogenous pluripotent genes were highly expressed in NC- and SCA3-iPSC lines (Figure 1(d)). These iPSC lines demonstrated normal karyotypes (Figure 1(e)) and formed teratoma presenting with tissues belonging to three germ layers (Figure 1(f)). Selected iPSC clones from each line (NC-1, NC-2, SCA3-1, and SCA3-2) with good 


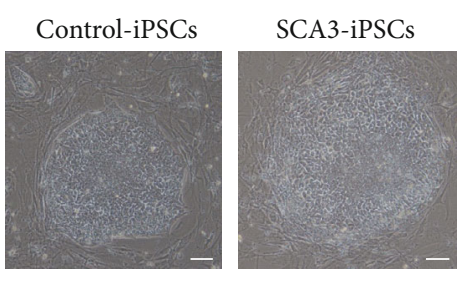

(a)
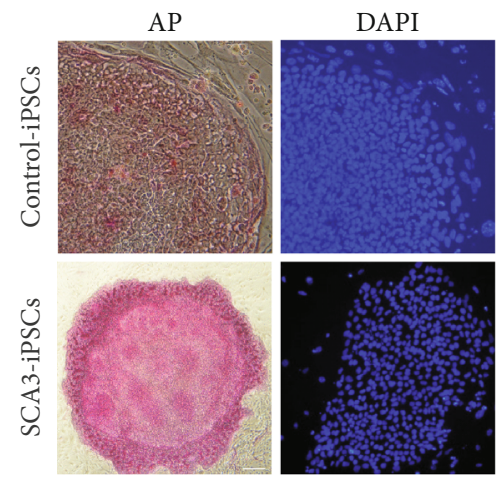

(c)

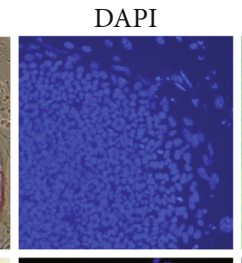

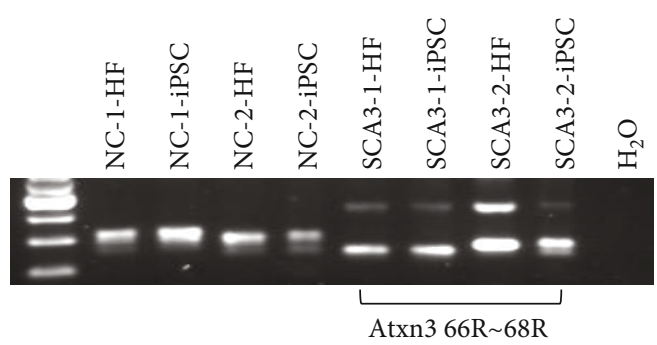

(b)

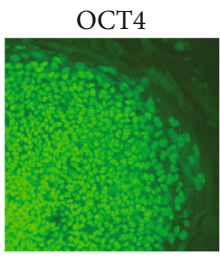

TRA-1-81
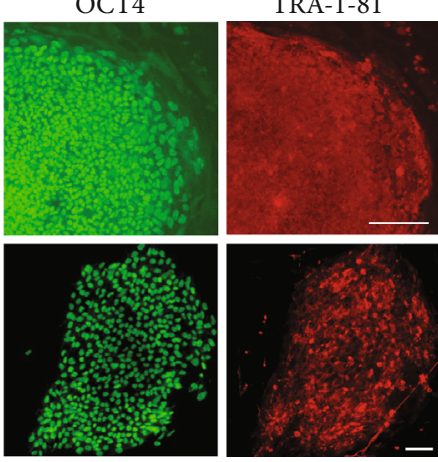

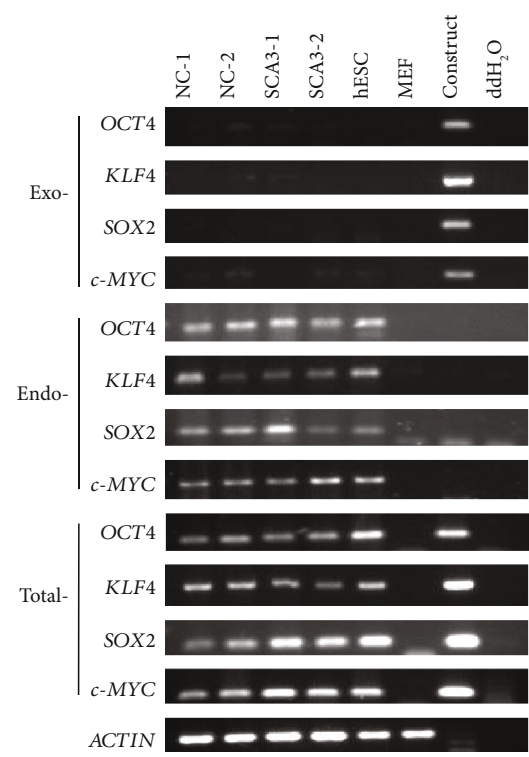

(d)

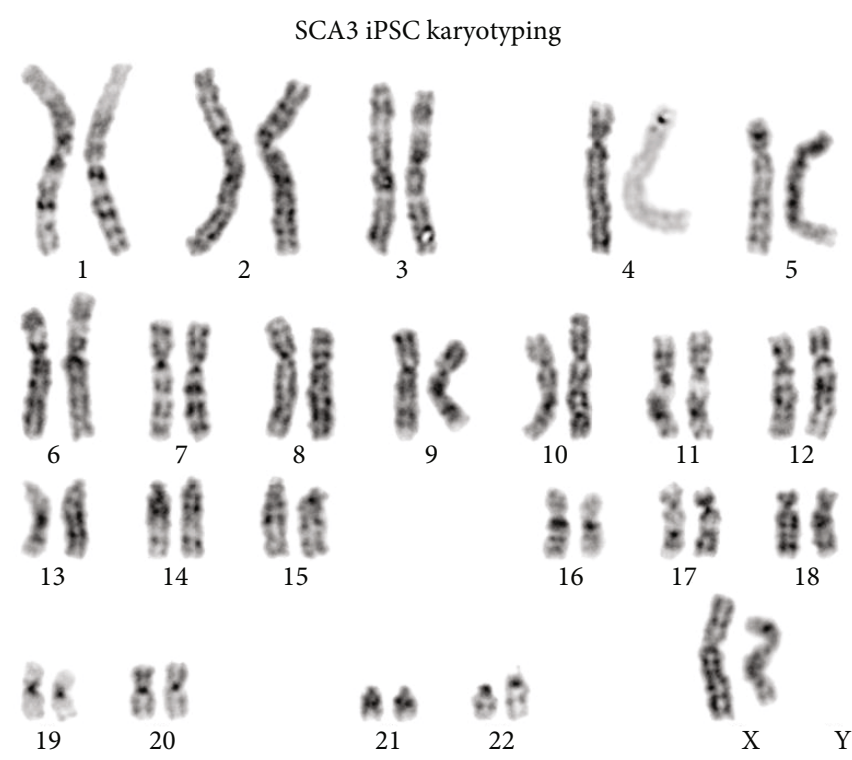

(e)

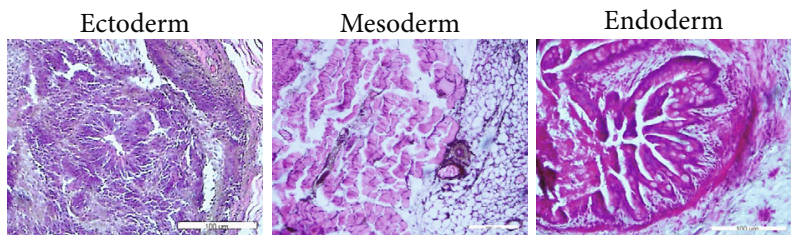

(f)

FIGURE 1: Characterization of SCA3 patient-specific-induced pluripotent stem cells (SCA3-iPSCs). (a) iPSC colonies obtained from reprogramming of normal controls and patients' fibroblasts. (b) Genotyping of iPSCs and fibroblasts (HF). PCR analysis showed SCA3 patients carrying expanded CAG repeats (66 68 repeats). (c) Immunofluorescent staining for the stem cell markers alkaline phosphatase (AP), OCT4 and TRA-1-81 in control- and SCA3-iPSCs. (d) RT-PCR analysis of exogenous and endogenous pluripotency markers (OCT4, KLF4, SOX2, and c-MYC genes) from iPSC cell lines. (e) Karyotyping analysis of SCA3-iPSCs. (f) Teratoma formed after subcutaneously injecting SCA3-iPSCs into nude mice. HE staining of teratomas showed endoderm, mesoderm, and ectoderm lineages. Scale bar: $100 \mu \mathrm{m}$. 
morphological features and absent expression of exogenous genes were used for further investigation.

Using a feeder-free monolayer differentiation protocol with defined chemicals (Figure 2(a)), these iPSCs became elongated and expressed NSC markers NESTIN and PAX6 four weeks after differentiation (Figure 2(b)). Mature neurons expressing TUJ1 were generated following another two weeks of differentiation (Figure 2(c)). The trypan blue exclusion test showed that neurons derived from SCA3- and NCiPSCs demonstrated similar cell viability (ranging from 94 to $96 \%, p>0.05$, Figure 2(d)). Caspase 3 activities of neurons were also similar between SCA3 patients and NC subjects (NC set as 100\%; NC-1 vs. SCA3-1: $100 \%$ vs. $109 \%, p>$ 0.05 ; NC-2 vs. SCA3-2: $100 \%$ vs. $93 \%, p>0.05$, Figure $2(\mathrm{e}))$.

3.2. Accumulation of PolyQ Aggregation in SCA3-iPSCDerived Neurons. To investigate whether SCA3-iPSCderived neurons form polyQ aggregation, we performed a filter trap assay, which displayed greater intensity of aggregates positive to $1 \mathrm{C} 2$ staining, a marker for protein aggregation [23], in SCA3-iPSC-derived neurons compared to NCiPSC-derived neurons (Figure 3(a)). Immunocytochemistry staining consistently showed a significant number of $1 \mathrm{C} 2$ positive aggregates in SCA3-iPSC-derived neurons, while these aggregates were not seen in NC-iPSC-derived neurons (Figure 3(b)). These 1C2-positive aggregates were located in the perinuclear region and also colocalized with P62, another marker for protein aggregation [24] (Figure 3(b)).

It has been shown that polyQ aggregation disturbed the UPS function [25]. The proteasome activity assay also showed that SCA3-iPSC-derived neurons had lower proteasome activity compared to NC-iPSC-derived neurons although the difference was not statistically significant (NC set as 100\%; NC-1 vs. SCA3-1: $100 \%$ vs. $92 \%, p=0.057$; NC-2 vs. SCA3-2: $100 \%$ vs. $93 \%, p=0.226$, Figure $3(\mathrm{c}))$.

3.3. Proteasome Dysfunction and Increased Oxidative Stress in MG132-Stimulated SCA3-iPSC-Derived Neurons. MG132 has been widely used as a potent proteasome inhibitor targeting $26 \mathrm{~S}$ proteasome to block its activity [26]. To reveal the potential protein degradation impairment in SCA3, neurons derived from iPSCs were treated with MG132 (0 5 $\mu \mathrm{M})$ for 24 hours and then harvested for the proteasome activity assay. Treatment with MG132 significantly reduced proteasome activity in a dose-dependent manner in all iPSCderived neurons (untreated set as 100\%; 0 5 $\mu \mathrm{M}$ MG132; NC-1: $100 \% \sim 53 \%, p=0.005 \sim<0.001$; SCA3-1: $100 \% \sim 39 \%$, $p<0.001$; NC-2: $100 \% \sim 50 \%, p=0.002 \sim 0.001$; SCA3-2: $100 \% \sim 38 \%, p=0.005 \sim<0.001$, Figure 4(a)). Gender-matched comparison showed that the reduction of proteasome activity was greater in SCA3-iPSC-derived neurons compared to NCiPSC-derived neurons (SCA3-1 vs. NC-1: $74 \%$ vs. $84 \%$ (0.5 $\mu \mathrm{M}$ MG132), 64\% vs. $72 \%$ ( $1 \mu \mathrm{M}$ MG132), $49 \%$ vs. $62 \%$ ( $2 \mu \mathrm{M}$ MG132), and $39 \%$ vs. 53\% (5 $\mu \mathrm{M}$ MG132), $p=$ $0.034 \sim 0.001$; SCA3-2 vs. NC-2: $67 \%$ vs. $81 \%(0.5 \mu \mathrm{M}$ MG132), $59 \%$ vs. $71 \%$ ( $1 \mu \mathrm{M}$ MG132), $55 \%$ vs. $60 \%(2 \mu \mathrm{M}$ MG132), and $38 \%$ vs. 50\% (5 $\mu$ M MG132), $p=0.049 \sim 0.014$, Figure $4(\mathrm{a}))$. The filter trap assay also detected pronounced aggregates following $2 \mu \mathrm{M}$ MG132 treatment in SCA3-
iPSC-derived neurons (Figure 4(b)). Treatment with $2 \mu \mathrm{M}$ MG132 specifically increased 1C2-positive aggregates in SCA3-iPSC-derived neurons, while the aggregation did not appear in NC-iPSC-derived neurons following MG132 treatment (Figure 4(c)). Treatment with $2 \mu \mathrm{M}$ MG132 further increased the amount of ROS in SCA3-iPSC-derived neurons compared to the NC-iPSC-derived neurons (Figure 4(d)).

\subsection{SCA3-iPSC-Derived Neurons Demonstrating Increased} Susceptibility to Proteasome Inhibitor. It has been reported that inhibition of proteasome function by MG132 induced apoptotic cell death in a Parkinson's disease (PD) iPSC model [20]. Using the LDH assay, we found that under $2 \mu \mathrm{M}$ MG132 treatment, SCA3-iPSC-derived neurons released a higher level of $\mathrm{LDH}$ compared to NC-iPSC-derived neurons (MG132untreated neurons set as $100 \%$, MG132-treated SCA3-1 vs. NC-1: $229 \%$ vs. $189 \%, p=0.020$; MG132-treated SCA3-2 vs. NC-2: $204 \%$ vs. $165 \%, p=0.018$, Figure $5(\mathrm{a})$ ). Treatment with $2 \mu \mathrm{M}$ MG132 led to greater elevation of cleaved caspase 3 levels in both SCA3-iPSC-derived neurons compared to NCiPSC-derived neurons (MG132-treated NC-1 and NC-2 set as 100\%; MG132-treated SCA3-1 vs. NC-1: $147 \%$ vs. $100 \%$, $p<0.001$; MG132-treated SCA3-2 vs. NC-2: $146 \%$ vs. $100 \%$, $p=0.040$, Figure 5(b)). $2 \mu \mathrm{M}$ MG132 treatment also more greatly upregulated caspase 3 activities in SCA3-iPSCderived neurons compared to NC-iPSC-derived neurons (MG132-untreated neurons set as $100 \%$, MG132-treated SCA3-1 vs. $\mathrm{NC}-1: 436 \%$ vs. $247 \%, p=0.002$; MG132treated SCA3-2 vs. NC-2: $451 \%$ vs. $278 \%, p=0.012$, Figure 5(c)). These results suggested that compared to the NC-iPSC-derived neurons, SCA3-iPSC-derived neurons were more susceptible to the MG132 treatment.

3.5. NH037 and Daidzein Enhancing Proteasome Function in iPSC-Derived Neurons. Previously, we found that Chinese herbal medicine $\mathrm{NH} 037$ and its constituent daidzein exerted neuroprotective effects by promoting proteasome function and reducing apoptotic protein expression in ATXN3/ ${ }_{75^{-}}$ GFP expressing 293/SH-SY5Y cells. Previously, we have found that the $\mathrm{IC}_{50}$ value of $\mathrm{NH} 037$ and daidzein was $>30 \mathrm{mg} / \mathrm{ml}$ and $100 \mu \mathrm{M}$ in ATXN3/Q 75 -GFP 293/SH-SY5Y cell models, suggesting low cytotoxicity of NH037 and daidzein [19]. Our previous study also showed that NH037 at $10 \mu \mathrm{g} / \mathrm{ml}$ and daidzein at $0.1 \mu \mathrm{M}$ displayed neuroprotective effects on ATXN3/Q ${ }_{75}$-GFP SH-SY5Y cells [19]. According to these results and our observation that an effective concentration of a compound used to treat iPSC-derived neurons could be much higher than SH-SY5Y cells, we administered $\mathrm{NH} 037$ at $1 \mathrm{mg} / \mathrm{ml}$ and daidzein at $50 \mu \mathrm{M}$ to iPSC-derived neurons. We found that NH037 $(1 \mathrm{mg} / \mathrm{ml})$ slightly enhanced proteasome activity in NC- but not SCA3iPSC-derived neurons (untreated set as 100\%; untreated vs. NH037: $100 \%$ vs. $107 \% \sim 118 \%, p=0.014 \sim 0.207$; untreated vs. daidzein: $100 \%$ vs. $109 \% \sim 118 \%, p=0.106 \sim 0.326$, Supplementary Figure 1(a)). Treatment with NH037 $(1 \mathrm{mg} / \mathrm{ml})$ or daidzein $(50 \mu \mathrm{M})$ rescued the downregulation of proteasome activities by $2 \mu \mathrm{M}$ MG132 in both NC- and SCA3-iPSCderived neurons (untreated set as 100\%; MG132 vs. MG132/NH037 or MG132/daidzein; NC-1: $48 \%$ vs. $90 \%$ or 


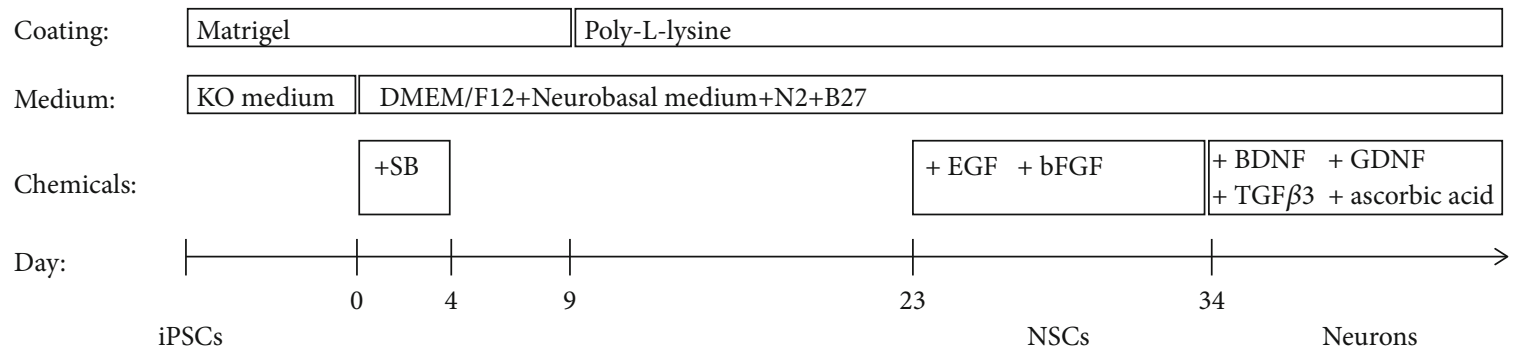

(a)
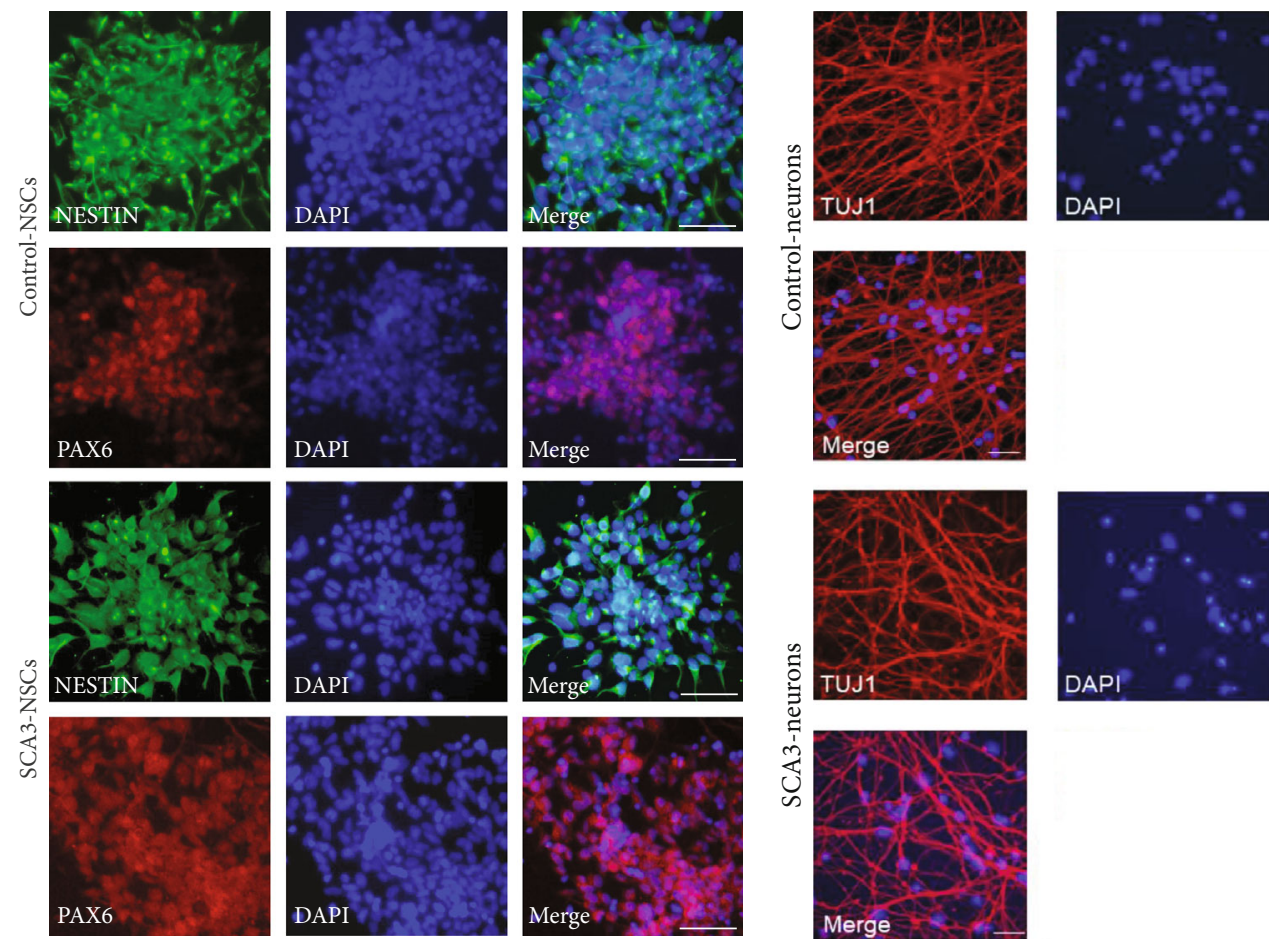

(b)

(c)
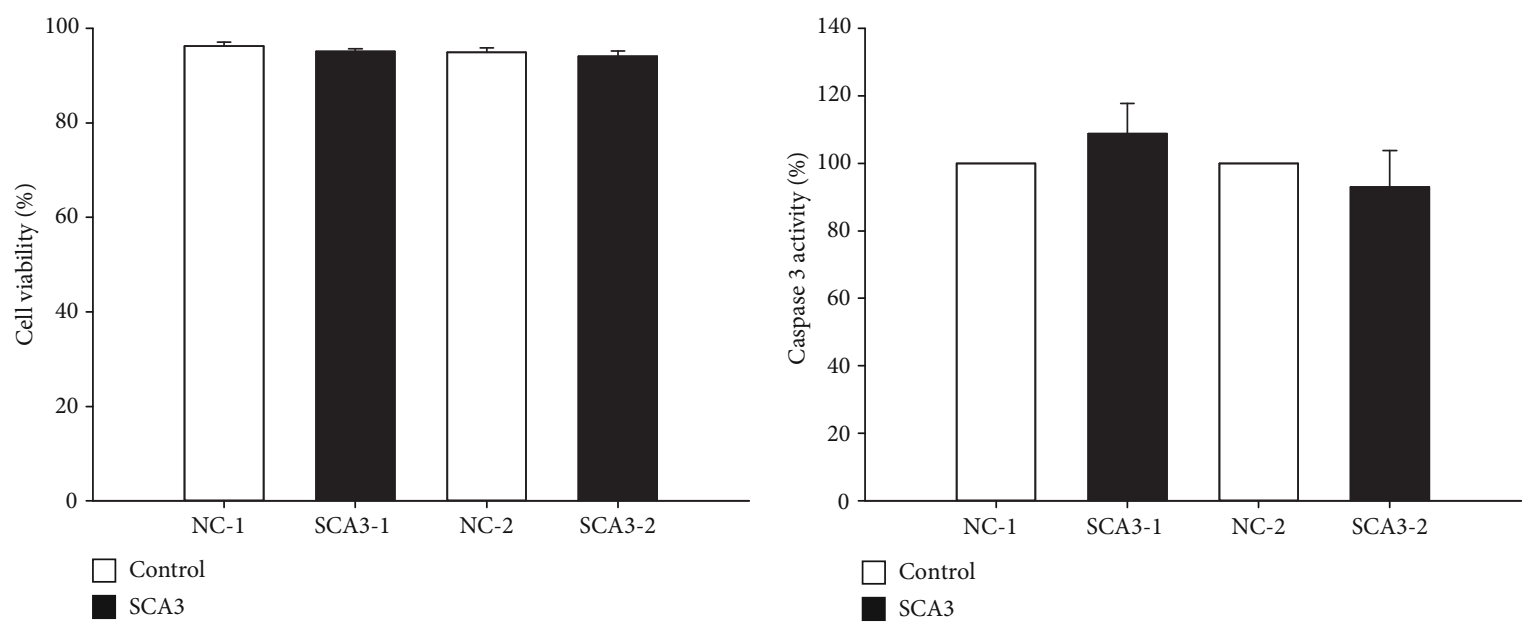

(d)

(e)

FIGURE 2: Differentiation of neural progenitors and neurons from control- and SCA3-iPSCs. (a) Flow chart of neuronal differentiation from iPSCs. KO: Knockout medium; SB: SB431542. (b) Neural stem cells (NSCs) positive to NESTIN (green) and PAX6 (red) were generated following three weeks of differentiation from iPSCs. (c) Matured neurons expressing TUJ1 (red) were obtained following another two weeks of differentiation from NSCs. Scale bar: $50 \mu \mathrm{m}$. (d) Cell viability was determined by the trypan blue exclusion assay, and (e) caspase 3 activity was examined in the control- (NC-) and SCA3-iPSC-derived neurons. The caspase 3 activity in NC-iPSC-derived (NC-1 and NC-2) neurons was normalized as $100 \%$. Each experiment for each sample was performed in triplicate. 
Filter trap assay

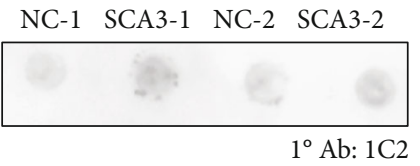

(a)
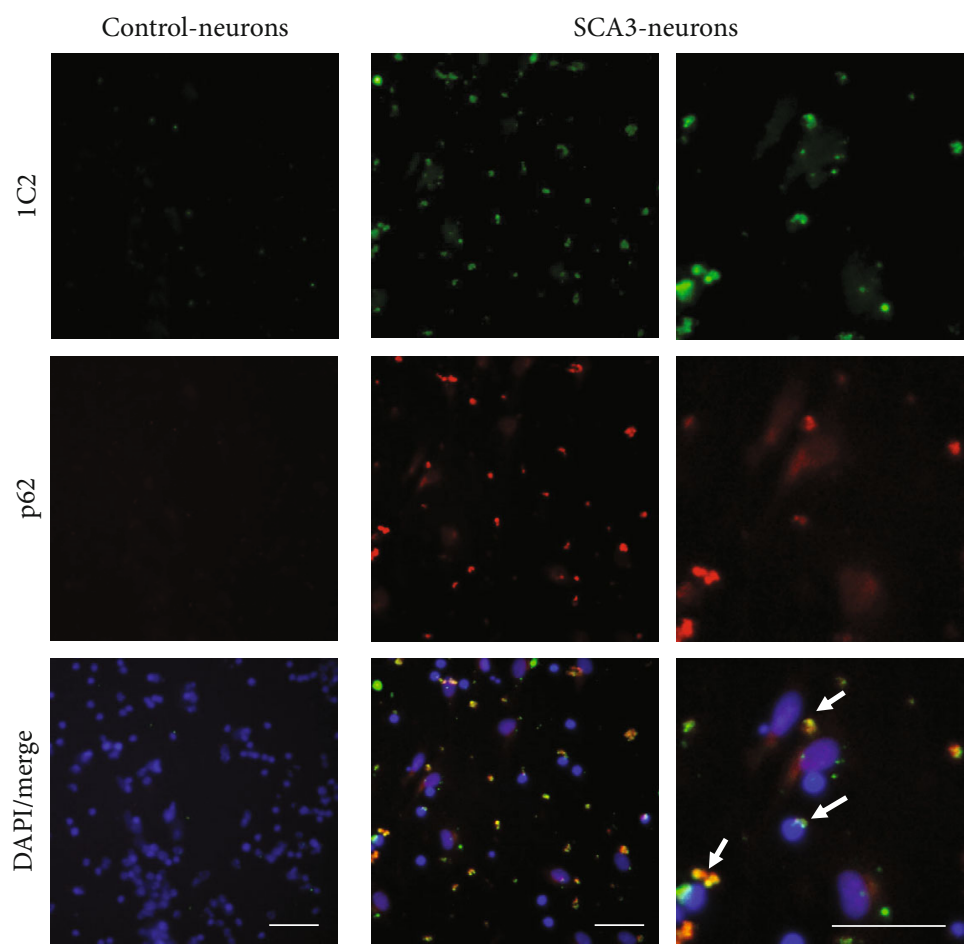

(b)

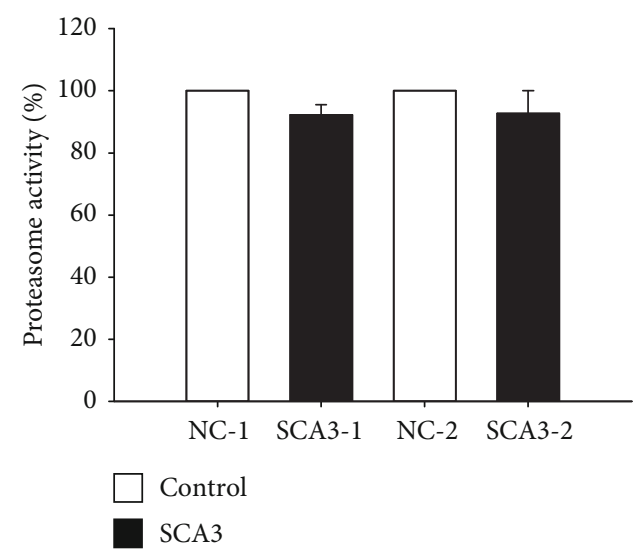

(c)

FIgURe 3: PolyQ aggregation and proteasome activity of SCA3-iPSC-derived neurons. (a) Filter trap assay was conducted with 1C2 antibody staining to detect the accumulation of polyQ aggregates. (b) SCA3-iPSC-derived neurons showed 1C2 staining-positive aggregates (green) and the aggregates colocalized with p62 (red) (white arrow). Scale bar: $50 \mu \mathrm{m}$. (c) Proteasome activity assay was performed to analyze UPS function in iPSC-derived neurons. NC-iPSC-derived neurons (NC-1 and NC-2) were normalized as 100\%. Each experiment for each sample was performed in triplicate.

$107 \%, p=0.005$ or 0.007 ; SCA3-1: $41 \%$ vs. $113 \%$ or $96 \%$, $p=0.001$ or 0.003 ; NC-2: $50 \%$ vs. $99 \%$ or $98 \%, p=0.018$ or 0.006 ; SCA3-2: $37 \%$ vs. $107 \%$ or $94 \%, p=0.010$ or 0.022 , Figure 6(a)). Treatment with $2 \mu \mathrm{M}$ MG132 produced a higher expression of smeared ubiquitinated proteins on both NC- and SCA3-iPSC-derived neurons (MG132treated NC-1 and NC-2 set as 100\%; MG132-treated vs. untreated; NC-1: $100 \%$ vs. $50 \%, p=0.010$; SCA3-1: $105 \%$ vs. $63 \%, p=0.004$; NC-2: $100 \%$ vs. $42 \%, p<0.001$; SCA3-2: $152 \%$ vs. $78 \%, p=0.018$, Figure $6(\mathrm{~b}))$. The ubiquitination in 


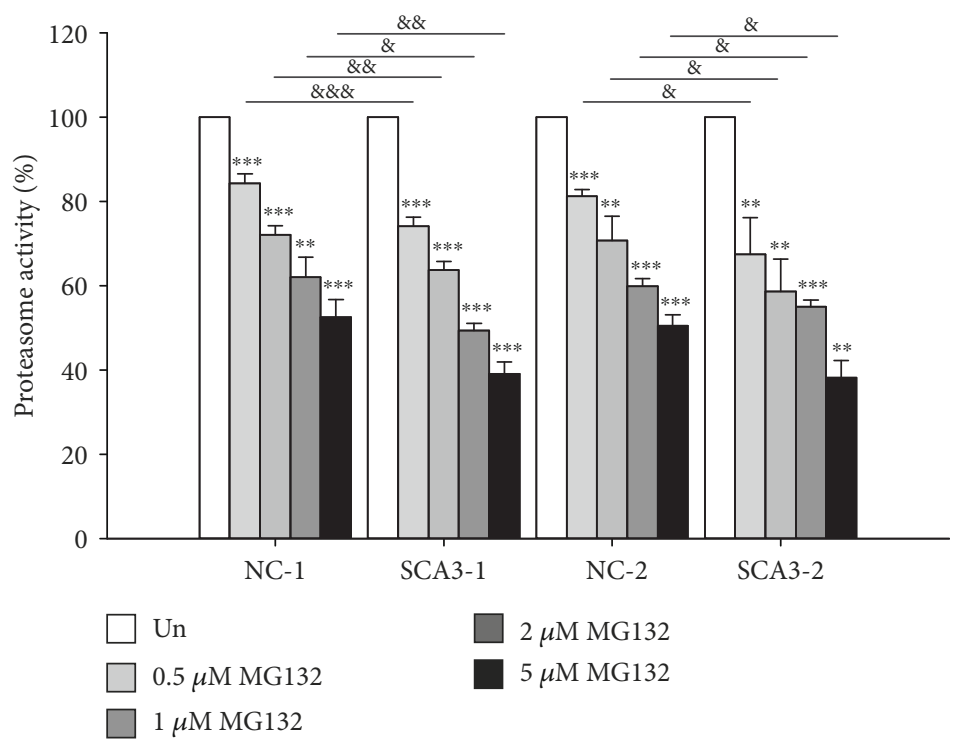

(a)

Filter trap assay

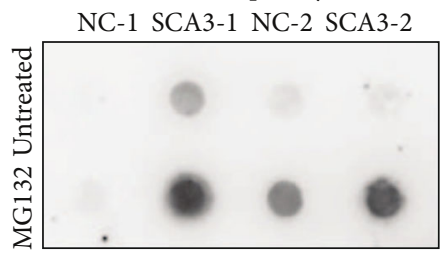

(b)
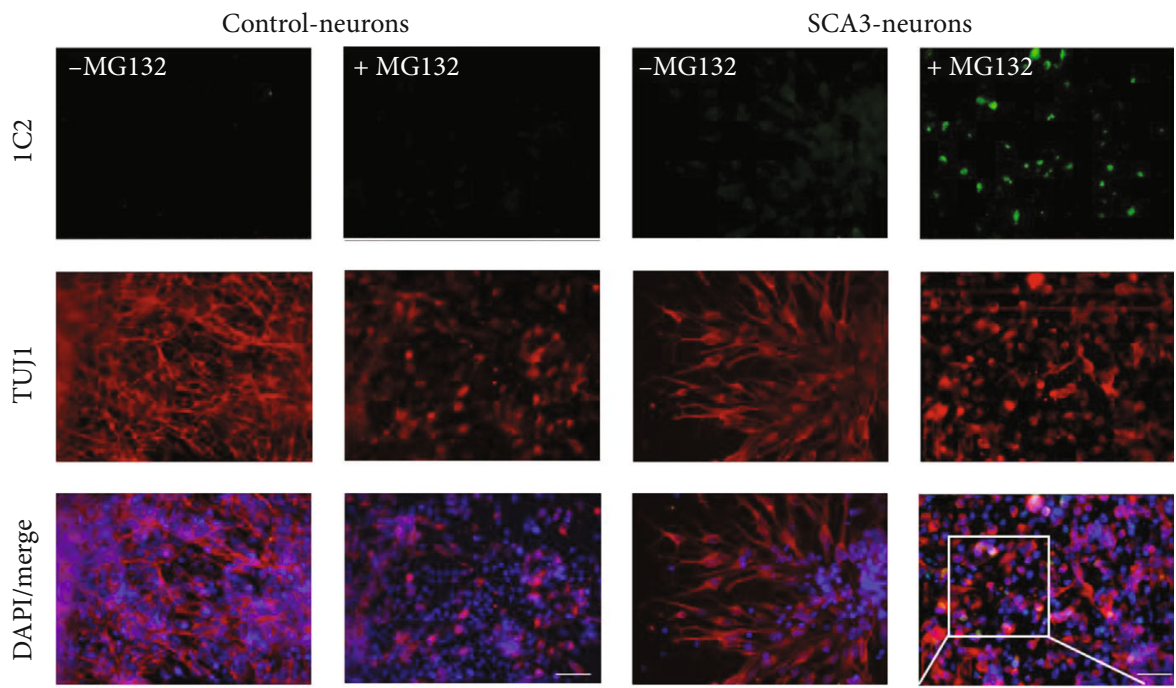

(c)

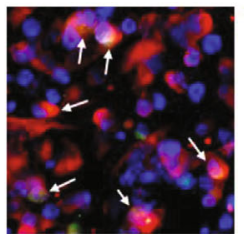

Figure 4: Continued. 

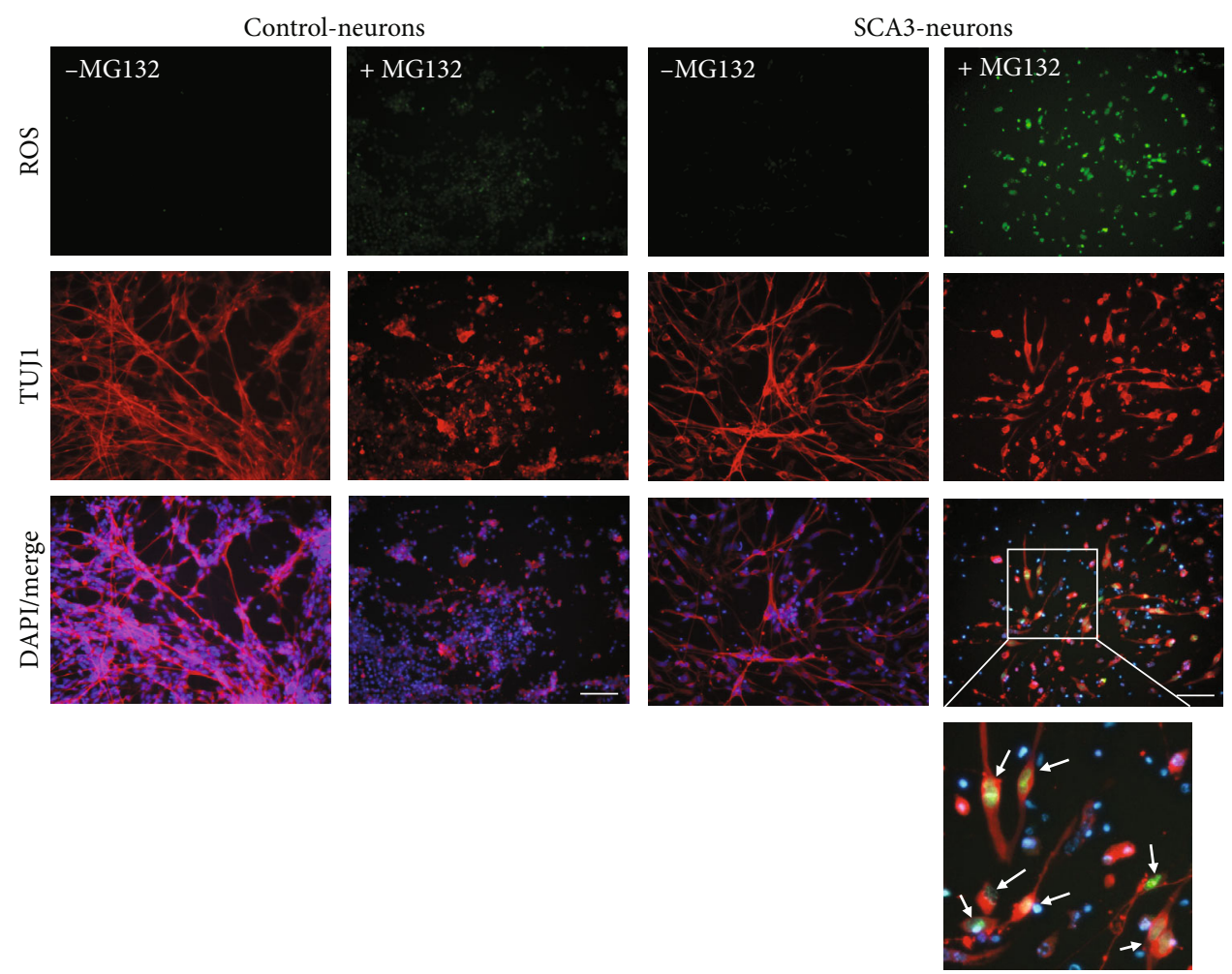

(d)

FIGURE 4: Impairment of the ubiquitin-proteasome system (UPS) in SCA3-iPSC-derived neurons treated with MG132. (a) Proteasome activity of iPSC-derived neurons treated with various concentrations of MG132 $(0 \sim 5 \mu \mathrm{M})$. Proteasome activity of untreated neurons was normalized as $100 \%$. (b) 1C2-positive polyQ aggregates in $2 \mu \mathrm{M}$ MG132-treated iPSC-derived neurons were detected by the filter trap assay. (c) Representative images of iPSC-derived neurons stained with 1C2 (green) and anti-TUJ1 (red). The white arrows in the magnified picture indicate 1C2-positive aggregates colocalized with TUJ1. (d) Representative images of iPSC-derived neurons stained with a cellular ROSdetecting reagent (green). The white arrows in the magnified picture indicate ROS detected in TUJ1-positive neurons. Scale bar: $50 \mu \mathrm{m}$. Each experiment for each sample was performed in triplicate. $p$ values: MG132-treated vs. untreated, ${ }^{* *} p<0.01$ and ${ }^{* * *} p<0.001$; SCA3-1 vs. NC-1 or SCA3-2 vs. NC-2, ${ }^{\&} p<0.05,{ }^{\& \&} p<0.01$, and ${ }^{\& \& \&} p<0.001$.

$2 \mu \mathrm{M}$ MG132-treated neurons was also reduced by the treatment with $1 \mathrm{mg} / \mathrm{ml} \mathrm{NH037} \mathrm{or} 50 \mu \mathrm{M}$ daidzein (MG132 vs. MG132/NH037 or MG132/daidzein; NC-1: $100 \%$ vs. $60 \%$ or $61 \%, p=0.017$ or 0.005 ; SCA3-1: $103 \%$ vs. $68 \%$ or $69 \%, p=0.002$ or 0.006 ; NC-2: $100 \%$ vs. $45 \%$ or $50 \%, p=$ 0.003 or $<0.001$; SCA3-2: $125 \%$ vs. $76 \%$ or $77 \%, p=0.002$ or 0.027 , Figure 6(b)).

In NC-iPSC-derived neurons, treatment with daidzein $(50 \mu \mathrm{M})$ and not NH037 $(1 \mathrm{mg} / \mathrm{ml})$ affects levels of MDA, a lipid peroxidation marker [27], but either NH037 or daidzein has no effect on SCA3-iPSC-derived neurons (untreated set as 100\%; untreated vs. NH037: $100 \%$ vs. $99 \% \sim 104 \%, p=$ 0.072 0.971; untreated vs. daidzein: $100 \%$ vs. $97 \% \sim 103 \%$, $p=0.004 \sim 0.401$, Supplementary Figure 1(b)). Treatment with $2 \mu \mathrm{M}$ MG132 further generated higher levels of MDA compared to untreated cells (untreated set as $100 \%$; MG132-treated: NC-1: $115 \%, p=0.021$; SCA3-1: $145 \%$, $p=0.006 ; \mathrm{NC}-2: 135 \%, p=0.014 ;$ SCA3-2: $155 \%, p=$ 0.002 , Figure $6(\mathrm{c})) .1 \mathrm{mg} / \mathrm{ml} \mathrm{NH037}$ or $50 \mu \mathrm{M}$ daidzein significantly normalized the MDA level under $2 \mu \mathrm{M}$ MG132 treatment (MG132 vs. MG132/NH037 or MG132/daidzein; NC-1: $115 \%$ vs. $101 \%$ or $97 \%, p=0.064$ or 0.005 ; SCA3-1:
$145 \%$ vs. $111 \%$ or $100 \%, p=0.009$ or 0.006 ; NC-2: $135 \%$ vs. $109 \%$ or $110 \%, p=0.007$ or 0.017 ; SCA3-2: $155 \%$ vs. $101 \%$ or $103 \%, p<0.001$ or $=0.025)$. Importantly, treatment with $1 \mathrm{mg} / \mathrm{ml} \mathrm{NH037}$ or $50 \mu \mathrm{M}$ daidzein significantly reduced ROS levels in SCA3-iPSC-derived neurons (Figure 6(d)).

3.6. NH037 and Daidzein Reducing Cytotoxicity in iPSCDerived Neurons. Treatment with NH037 (1 mg/ml) and not daidzein $(50 \mu \mathrm{M})$ slightly decreased release of LDH in SCA3-iPSC-derived neurons, whereas either NH037 or daidzein has no effect on NC-iPSC-derived neurons (untreated set as 100\%; untreated vs. NH037: $100 \%$ vs. $83 \% \sim 90 \%, p=$ $0.004 \sim 0.381$; untreated vs. daidzein: $100 \%$ vs. $97 \% \sim 104 \%$, $p=0.612 \sim 0.949$, Supplementary Figure 2(a)). Treatment with $1 \mathrm{mg} / \mathrm{ml} \mathrm{NH037}$ or $50 \mu \mathrm{M}$ daidzein significantly ameliorated the $2 \mu \mathrm{M}$ MG132-induced abnormal release of $\mathrm{LDH}$ in both NC- and SCA3-iPSC-derived neurons (untreated set as 100\%; MG132 vs. MG132/NH037 or MG132/daidzein; NC-1: $164 \%$ vs. $115 \%$ or $113 \%, p=0.026$ or 0.024 ; SCA3-1: $218 \%$ vs. $144 \%$ or $155 \%, p=0.025$ or 0.094 ; NC-2: $166 \%$ vs. $124 \%$ or $134 \%, p=0.016$ or 0.038 ; SCA3-2: $196 \%$ vs. $134 \%$ or $120 \%$, $p=0.002$ or $<0.001$, Figure $7(\mathrm{a}))$. 

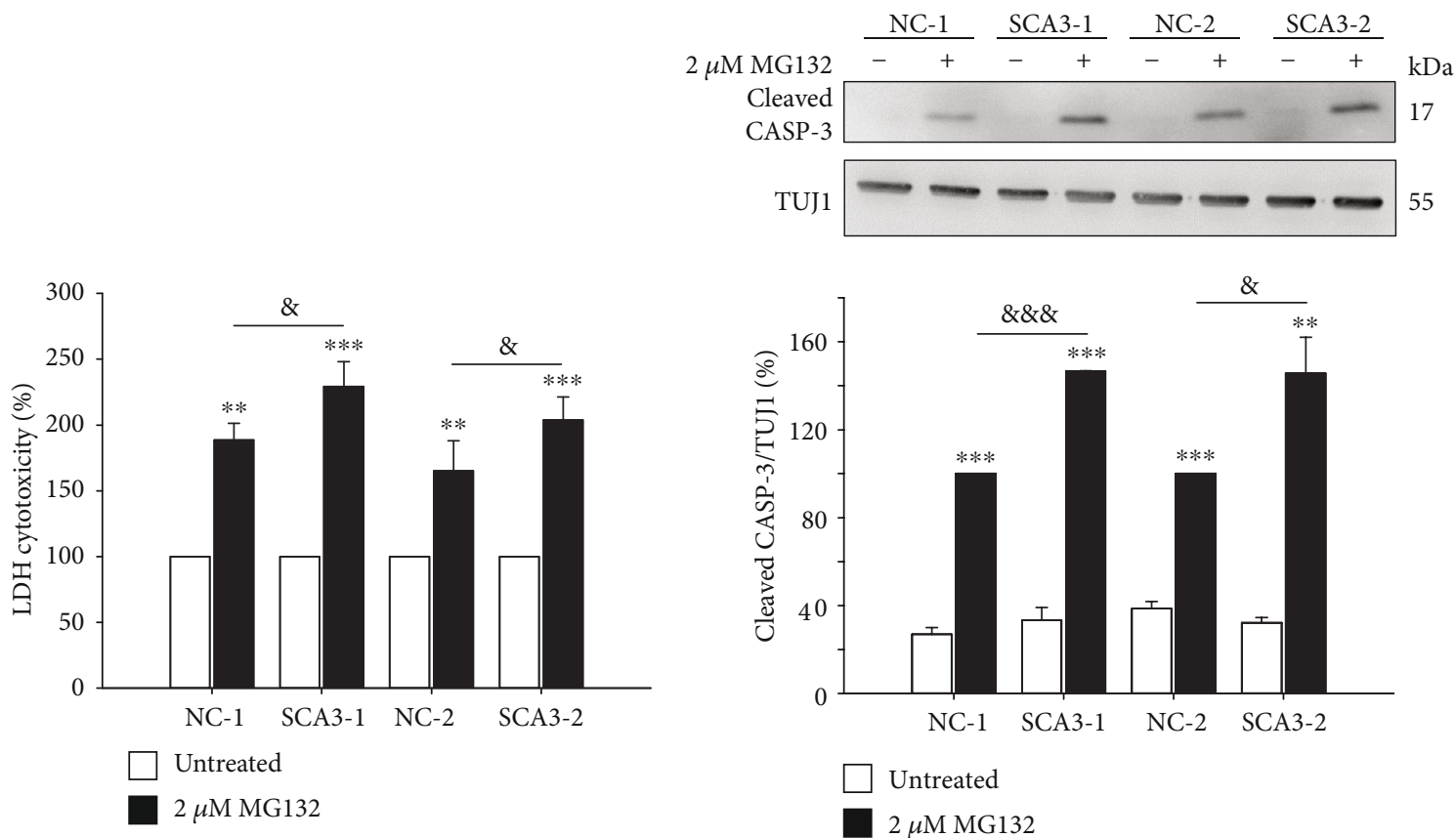

(a)

(b)

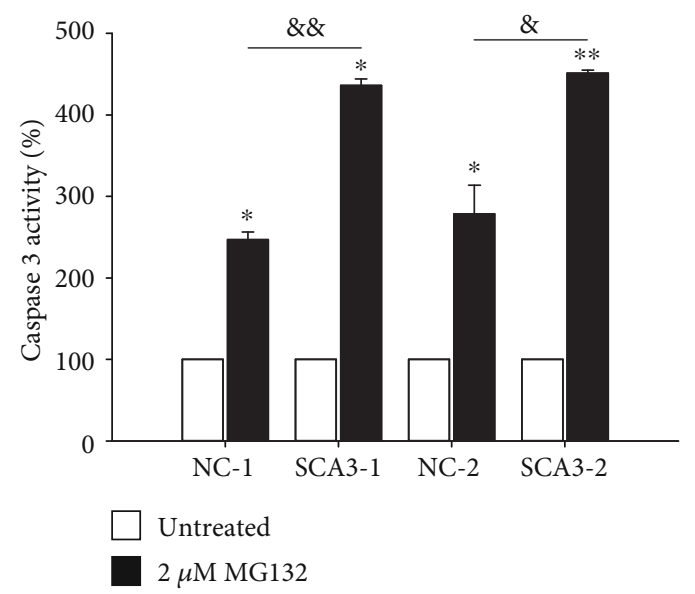

(c)

FIGURE 5: Elevated cytotoxicity and caspase 3 expression/activity in MG132-treated iPSC-derived neurons. (a) Lactic dehydrogenase (LDH) release in culture media was measured to evaluate cytotoxicity. LDH level of untreated neurons was normalized as $100 \%$. (b) Western blot analysis of the cleaved caspase 3 level (normalized to TUJ1 as the internal control). Cleaved caspase 3 level of $2 \mu \mathrm{M}$ MG132-treated NC-iPSCderived neurons (NC-1 and NC-2) was set as 100\%. (c) Caspase 3 activity assay of NC- and SCA3-iPSC-derived neurons. Caspase 3 activity of untreated neurons was normalized as $100 \%$. Each experiment for each sample was performed in triplicate. $p$ values: MG132-treated vs. untreated, ${ }^{*} p<0.05,{ }^{* *} p<0.01$, and ${ }^{* * *} p<0.001$; SCA3-1 vs. NC-1 or SCA3-2 vs. NC-2, ${ }^{\&} p<0.05,{ }^{\& \&} p<0.01$, and ${ }^{\& \& \&} p<0.001$.

$2 \mu \mathrm{M}$ MG132-induced upregulation of cleaved caspase 3 was also reduced by treatment with $1 \mathrm{mg} / \mathrm{ml} \mathrm{NH037}$ or $50 \mu \mathrm{M}$ daidzein (MG132-treated NC-1 and NC-2 set as 100\%; MG132 vs. MG132/NH037 or MG132/daidzein; NC-1: $100 \%$ vs. $64 \%$ or $84 \%, p=0.003$ or 0.088 ; SCA3-1: $134 \%$ vs. $62 \%$ or $62 \%, p=0.001$ or 0.003 ; NC-2: $100 \%$ vs. $61 \%$ or $69 \%, p=0.003$ or 0.014 ; SCA3-2: $135 \%$ vs. $101 \%$ or $71 \%, p=0.015$ or 0.001 , Figure $7(b))$. Treatment with $1 \mathrm{mg} / \mathrm{ml} \mathrm{NH037}$ and not $50 \mu \mathrm{M}$ daidzein slightly affects caspase 3 activity in SCA3-iPSC-derived neurons, whereas either $\mathrm{NH} 037$ or daidzein has no effect on NC-
iPSC-derived neurons (untreated set as $100 \%$; untreated vs. NH037: $100 \%$ vs. $87 \% \sim 106 \%, p=0.003 \sim 0.684$; untreated vs. daidzein: $100 \%$ vs. $101 \% \sim 113 \%, p=0.189 \sim 0.978$, Supplementary Figure 2(b)). Treatment of $1 \mathrm{mg} / \mathrm{ml} \mathrm{NH037} \mathrm{significantly}$ decreased MG132-induced upregulation of caspase 3 activity specifically in SCA3-iPSC-derived neurons (untreated set as $100 \%$; MG132 vs. MG132/NH037; SCA3-1: $442 \%$ vs. $344 \%$, $p=0.016$; SCA3-2: $365 \%$ vs. $200 \%, p=0.004$ ), while $50 \mu \mathrm{M}$ daidzein reduced caspase 3 activity only in SCA3-2-derived neurons (untreated set as 100\%, MG132 vs. MG132/daidzein; $365 \%$ vs. $212 \%, p=0.020$ ) (Figure $7(\mathrm{c})$ ). 

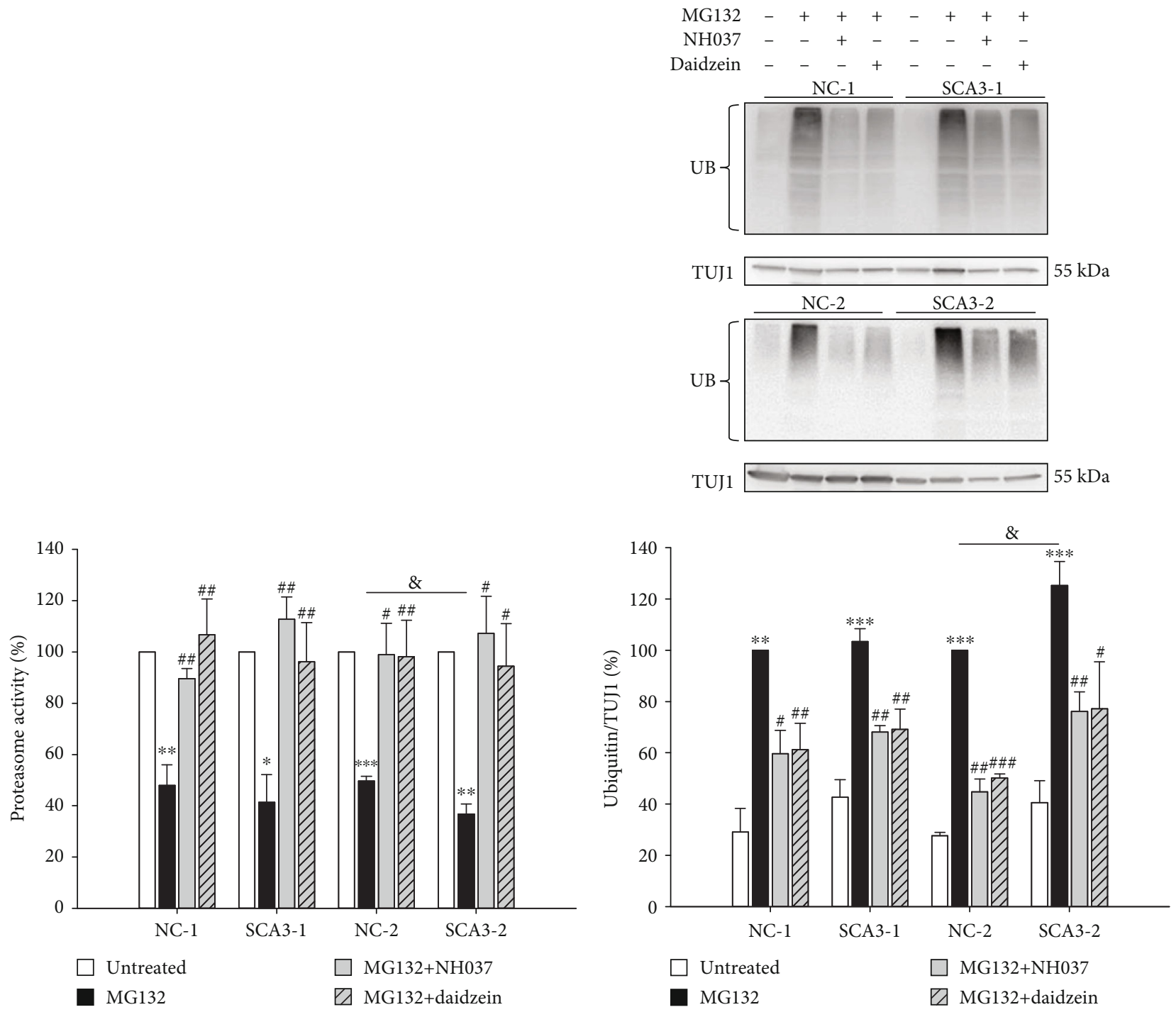

(a)

(b)

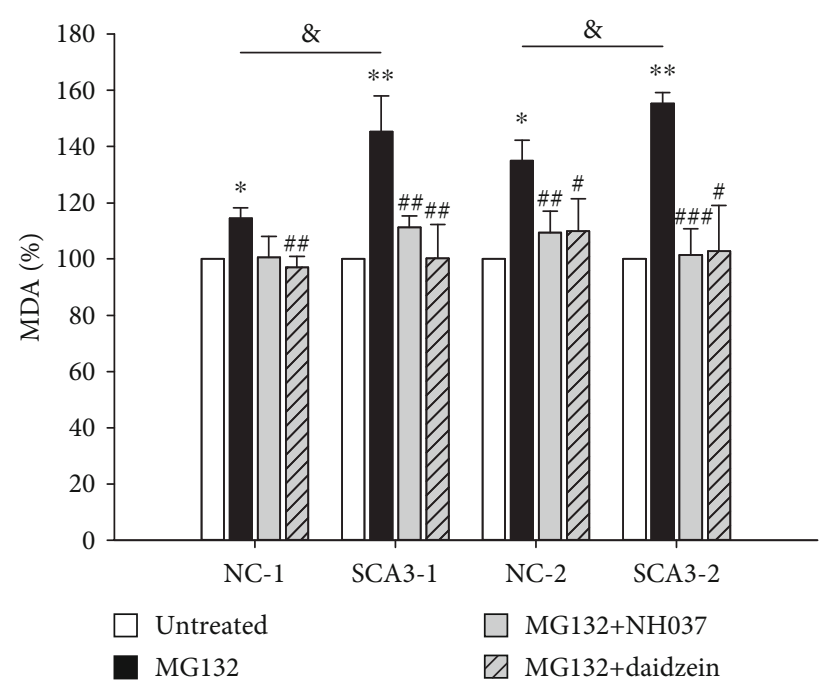

(c)

Figure 6: Continued. 

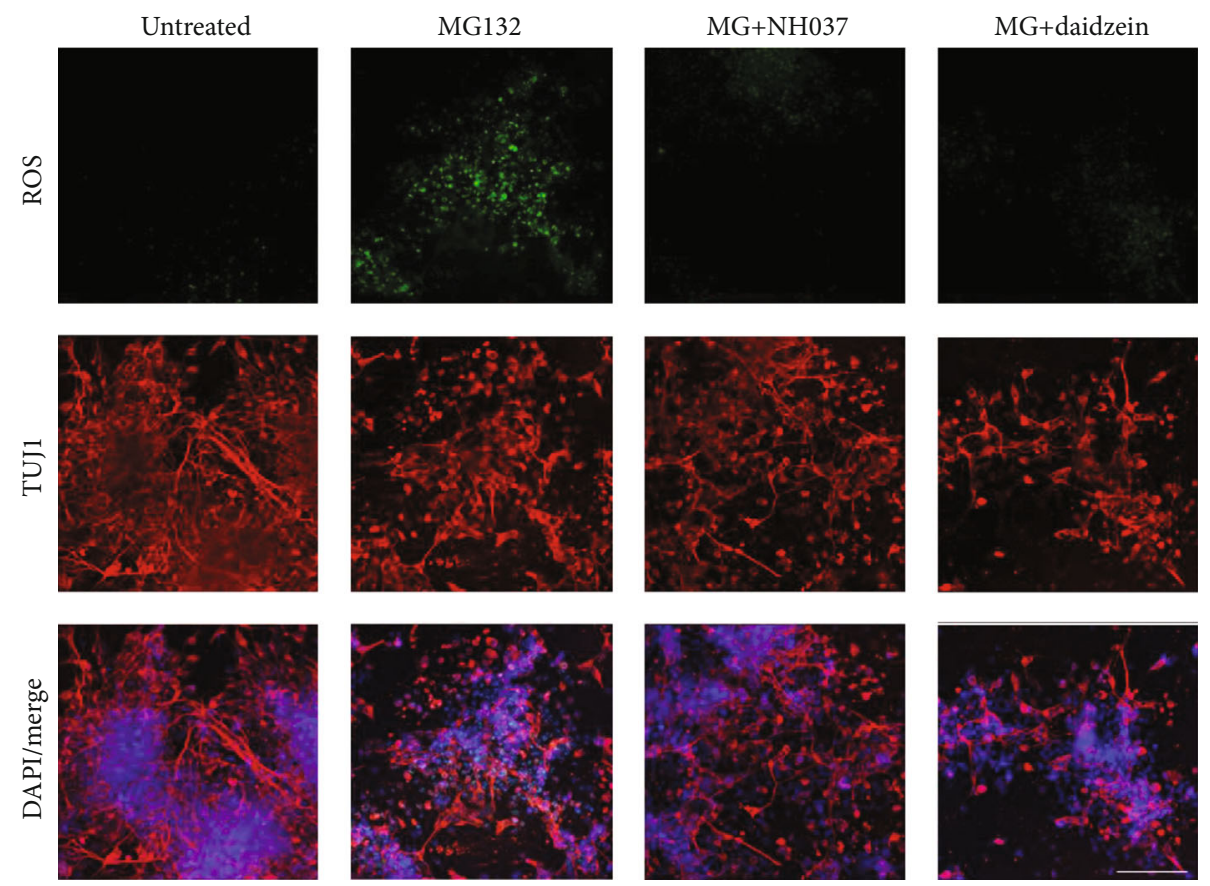

(d)

FIGURE 6: NH037 and daidzein promoted proteasome function and ameliorated oxidative stress in MG132-treated SCA3-iPSC-derived neurons. (a) Proteasome activity of iPSC-derived neurons treated with $2 \mu \mathrm{M}$ MG132 alone or MG132 together with $1 \mathrm{mg} / \mathrm{ml}$ NH037 or $50 \mu \mathrm{M}$ daidzein. The proteasome activity of untreated neurons was normalized as $100 \%$. (b) Western blot analysis of ubiquitin. TUJ1 was used as the internal control. Ubiquitinated protein level of MG132-treated NC-iPSC-derived neurons (NC-1 and NC-2) was set as 100\%. (c) Lipid peroxidation malondialdehyde (MDA) assay was conducted to evaluate oxidative stress. MDA level of untreated neurons was normalized as 100\%. (d) Representative images of SCA3 neurons staining with TUJ1 (red) and cellular ROS detecting reagent (green) after treatment with $2 \mu \mathrm{M}$ MG132, $1 \mathrm{mg} / \mathrm{ml} \mathrm{NH037,} \mathrm{or} 50 \mu \mathrm{M}$ daidzein. Scale bar: $50 \mu \mathrm{m}$. Each experiment for each sample was performed in triplicate. $p$ values: MG132-treated vs. untreated, ${ }^{*} p<0.05,{ }^{* *} p<0.01$, and ${ }^{* * *} p<0.001$; SCA3-1 vs. NC-1 or SCA3-2 vs. NC-2, ${ }^{\&} p<0.05$; MG132/NH037 vs. MG132 alone or MG132/daidzein vs. MG132 alone, ${ }^{\#} p<0.05,{ }^{\# \#} p<0.01$, and ${ }^{\# \# \#} p<0.001$.

\section{Discussion}

In this study, we successfully established iPSC lines and iPSC-derived neurons from patients with SCA3 (Figures 1 and 2). SCA3-iPSC-derived neurons showed abnormal intracellular aggregates, decreased proteasome function, pronounced ROS, and prominent cytotoxicity under proteasome inhibition by MG132 (Figures 3-5). NH037 or daidzein provided neuroprotection against MG132 cytotoxicity through enhancing UPS and suppressing the production of ROS on SCA3-iPSC-derived neurons (Figures 6-8). These findings indicate an important role of gene-environmental interaction in SCA3-mediated neurodegeneration, as well as the potential of proteasome enhancer in treating SCA3.

The rapid development in the iPSC field and the potential of iPSC to differentiate into different cell types not only offer great opportunities to study the pathogenesis of neurodegenerative diseases including SCA3 but also provide a platform to identify new therapeutic strategies [28-31]. In the past decade, neuronal cells derived from patient-specific iPSCs have been generated to model neurodegenerative diseases such as PD [32, 33], Alzheimer's disease (AD) [34, 35], and HD [36, 37], and relatively handful iPSC studies focused on cerebellar ataxias including Friedreich's ataxia (FRDA) [38-43] and SCAs: SCA2 [44], SCA3 [45-48], SCA6 [49],
SCA7 [50], SCA14 [51], and SCA36 [52]. Koch et al. first reported that L-glutamate-induced excitation of SCA3iPSC-derived neurons initiated calpain-dependent proteolysis of ATXN3 followed by the formation of SDS-insoluble aggregates [46]. However, glutamate-induced ATXN3 aggregation was not observed in SCA3-iPSC-derived neurons generated by other groups using different differentiation protocols $[45,48]$. A high concentration of db-cAMP in their neuronal differentiation medium may reduce the formation of aggregates by increasing proteasome activity $[45,53]$. We performed neural differentiation of iPSCs without dbcAMP treatment, and 1C2-positive aggregates were detected in SCA3-iPSC-derived neurons (Figure 3(b)). Consistent with pervious findings $[46,48]$, SCA3- and control-iPSCderived neurons displayed similar cell viability without MG132 treatment (Figure 2(d)). However, treating neurons with MG132 resulted in lower cell viability, higher caspase 3 activity, impaired UPS function, and pronounced ROS in SCA3-iPSC-derived neurons (Figures 4 and 5), suggesting the important role of environmental stress in ATXN3related neurodegeneration. The gene-environmental interactions have been recapitulated by different iPSC models for neurodegenerative diseases. Treatment with MG132 has been reported to induce cell death in iPSC-derived neurons carrying PARKIN mutation for PD [20]. Stressors such as cytokine 


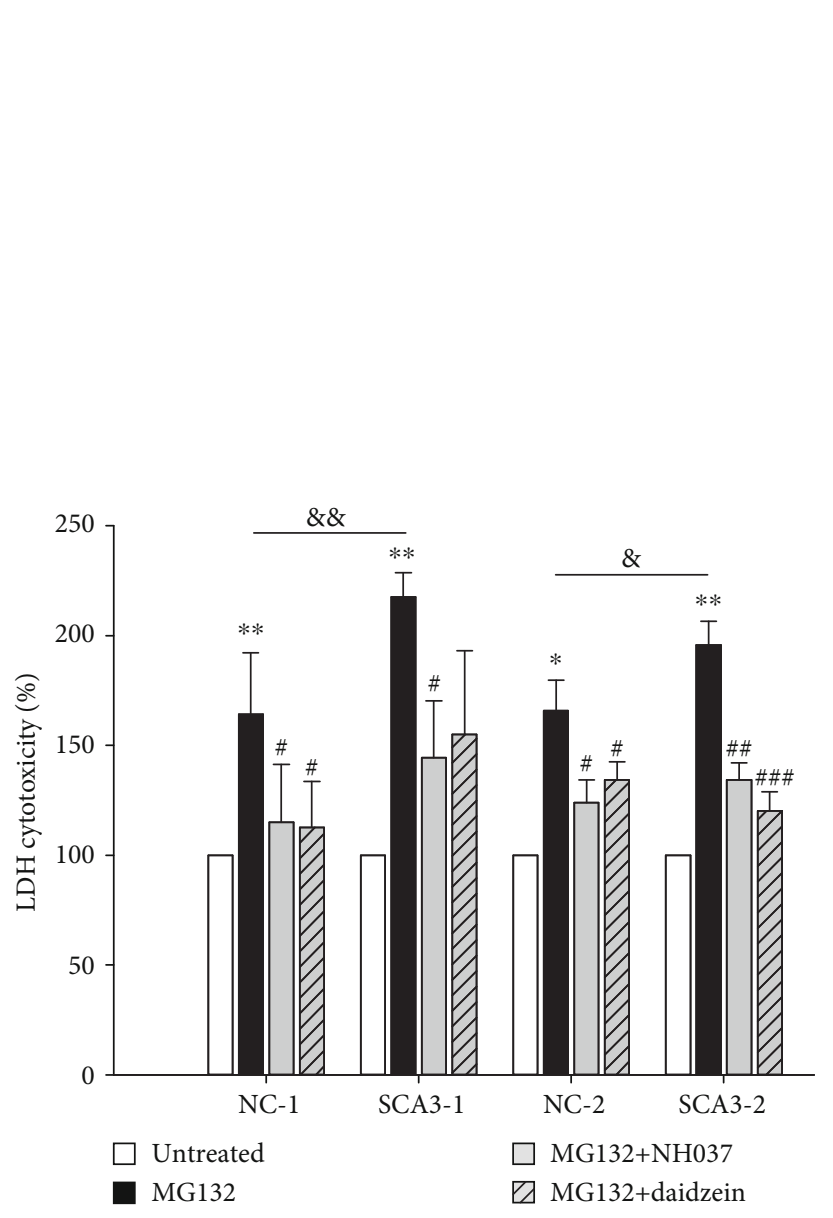

(a)
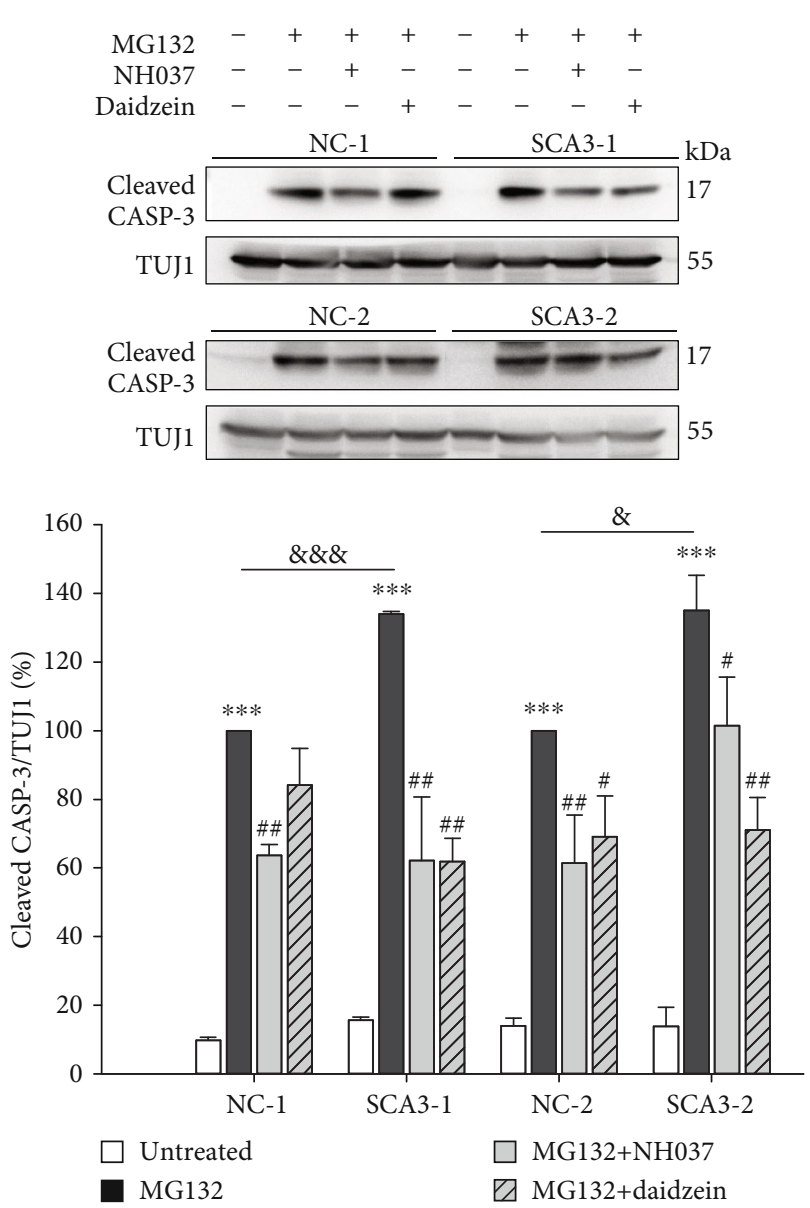

(b)

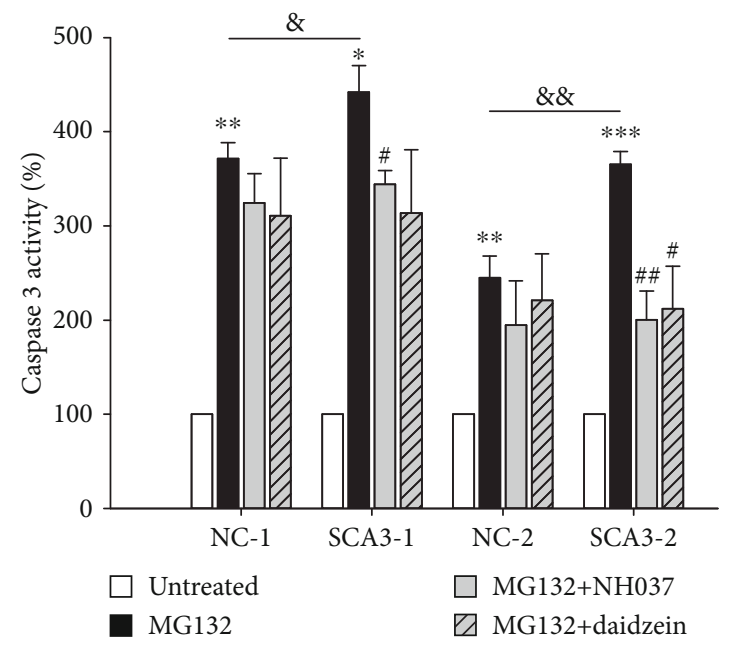

(c)

FIGURE 7: NH037 and daidzein reduced cytotoxicity and caspase 3 expression/activity in MG132-treated SCA3-iPSC-derived neurons. (a) LDH assay in iPSC-derived neurons treated with $2 \mu \mathrm{M}$ MG132 alone or MG132 and $1 \mathrm{mg} / \mathrm{ml} \mathrm{NH037/50} \mu \mathrm{M}$ daidzein (untreated neurons were normalized as 100\%). (b) Western blot analysis of cleaved caspase 3 (normalized to TUJ1 as the internal control) in iPSC-derived

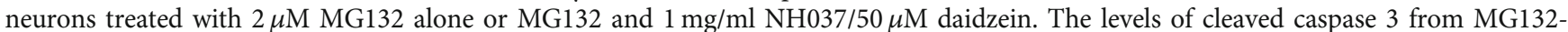
treated NC-iPSC-derived neurons (NC-1 and NC-2) were set as $100 \%$. (c) Caspase 3 activity in iPSC-derived neurons treated with $2 \mu \mathrm{M}$

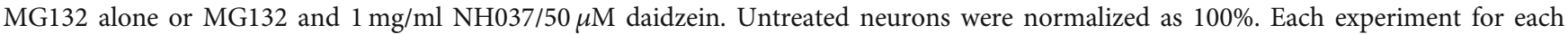
sample was performed in triplicate. $p$ values: MG132-treated vs. untreated, ${ }^{*} p<0.05,{ }^{* *} p<0.01$, and ${ }^{* * *} p<0.001$; SCA3-1 vs. NC-1 or SCA3-2 vs. NC-2, ${ }^{\&} p<0.05,{ }^{\& \&} p<0.01$, and ${ }^{\& \& \&} p<0.001$; MG132/NH037 vs. MG132 alone or MG132/daidzein vs. MG132 alone, ${ }^{\#} p<0.05,{ }^{\# \#} p<0.01$. 


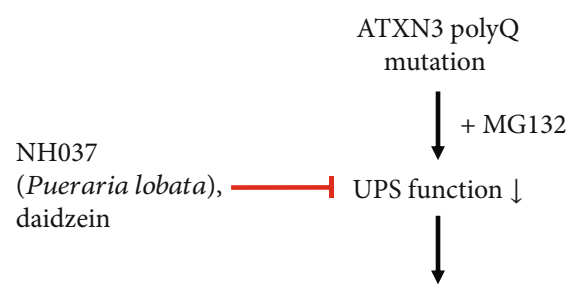

Ub-proteins $\uparrow$ PolyQ aggregation $\uparrow$

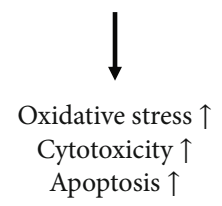

FIgURE 8: Hypothesis of NH037 and daidzein targeting UPS function in SCA3-iPSC-derived neurons. In SCA3-iPSC-derived neurons, UPS function is impaired under environmental stress (such as MG132 exposure), and ubiquitinated proteins and polyQexpanded ATXN3 protein are accumulated in cells. The resulting abnormal aggregation increased the production of oxidative stress and cytotoxicity. NH037 and daidzein promote protein degradation to reduce oxidative stress and cytotoxicity by improving proteasome activity in SCA3-iPSC-derived neurons.

treatment, oxidative stress, proteasome inhibition, or growth factor withdrawal are mandatory to generate phenotypes such as cell death, caspase 3 activation, or aggregate formation in HD-iPSC models [54-59]. Nutrient-depletion stress induced degeneration of dendrites in the iPSC-derived neurons from SCA6 patients [49]. These studies including our findings suggest the pivotal roles of environmental triggers in neurodegeneration. In this study, MG132 was used to trigger more aggregation, oxidative stress, and cytotoxicity in SCA3-iPSC-derived neurons, but proteasome inhibition could activate autophagy as a compensatory reaction during the cell recovery process $[60,61]$. Since our study did not examine the markers of autophagy, whether MG132induced activation of autophagy occurs in the SCA3-iPSCderived neurons remains to be clarified. It is worthy to mention that MG132 treatment on SCA3-iPSC-derived neurons still exacerbates aggregation, oxidative stress, and apoptosis, suggesting that even if there is an autophagy induction, it is not able to overcome the cytotoxicity induced by proteasome inhibition. In addition, although our previous study has shown no effect of NH037 and daidzein on autophagy in 293 cells [19], the autophagy-enhancing activity by NH037 and daidzein in our SCA3-iPSC model could not be excluded. Therefore, it is important to investigate if NH037 and/or daidzein also exert neuroprotection via acting on autophagy in SCA3-iPSC-derived neurons in the future.

While iPSC models provided insights into the pathogenesis of diseases, several studies have examined therapeutic applications in SCA3-iPSC models. Autophagy inducer, rapamycin, promoted autophagy and degraded expanded polyQ ATXN3 in neurons differentiated from SCA3-iPSCs [47]. Formation of SDS-insoluble aggregates in SCA3derived neurons can be suppressed by calpain inhibition [46]. However, none of these treatments demonstrated neu- roprotective effects on SCA3-iPSC-derived neurons. Our study, for the first time, demonstrates neuroprotective potentials of NH037 or daidzein by enhancing UPS to suppress oxidative stress, cytotoxicity, and apoptosis (Figures 6 and 7).

$\mathrm{NH} 037$ is an herbal extract prepared from $P$. lobata that is used traditionally to treat diarrhea, muscle stiffness, thirst, and diabetes in East Asia, and P. lobata has also been studied in several diseases including neurodegenerative diseases [62]. The main active constituents of $P$. lobata are isoflavonoids, which exhibit a wide range of biological activities including anti-inflammatory, antithrombotic, antihypertensive, antiarrhythmic, spasmolytic, and cancer chemopreventive properties [63]. Isoflavonoids also have demonstrated prominent neuroprotective effects against cerebrovascular disorders, hypertension, or PD in rats or cell models [64-66]. One of its most abundant isoflavonoids, daidzein [67, 68], exerts neuroprotective effect to enhance neuronal survival and has been implicated to be a potential drug for neurodegenerative diseases [69-71]. Treatment with daidzein in PC12 cells reduced $A \beta$ aggregates and the neuronal cytotoxicity of $A \beta$ [69]. In primary rat dorsal root ganglion (DRG) neuronal cultures, daidzein enhanced neuritogenesis depending on Src kinase, PKC $\delta$, and ERK signaling pathway [71]. Daidzein also improved neuronal cell viability and proliferation in vitro through activating the BDNF-TRK pathway [70]. In the SCA3-iPSC model, our study showed the neuroprotective effect of daidzein mediated by enhancing UPS, further indicating its pleiotropic effects on protecting neurons against different neurodegenerative pathogenesis.

\section{Conclusions}

In conclusion, this is the first study demonstrating UPS impairment in SCA3-iPSC-derived neurons and the neuroprotective potentials of NH037 and its constituent daidzein in SCA3. Recently, CRISPR/Cas9 genomic editing has been used to successfully eliminate the expanded polyQ in SCA3 patient-derived iPSCs [48]. It would be important to test the effects of NH037 and daidzein on SCA3-iPSC-derived and CRISPR/Cas9-corrected isogenic SCA3-iPSC-derived neurons to eliminate the variations caused by different genetic backgrounds. The acquisition of Purkinje cells from SCA3-iPSCs will further elucidate how the mutant ATXN3 selectively causes cerebellar degeneration [72]. Nevertheless, our findings shed lights on identification of new therapeutic compounds targeting UPS in SCA3. Further validation using various animal models will be warranted before translating our findings to clinical studies.

\section{Abbreviations}

AD: $\quad$ Alzheimer's disease

CHM: Chinese herbal medicine

DMEM: Dulbecco's modified Eagle medium

DRG: Dorsal root ganglion

FRDA: Friedreich's ataxia

HD: Huntington's disease

iPSC: Induced pluripotent stem cell

KSR: Knockout Serum Replacement 
LDH: Lactate dehydrogenase

MDA: Malondialdehyde

MEFs: Mouse embryonic fibroblasts

MJD: $\quad$ Machado-Joseph disease

NSC: $\quad$ Neural stem cell

PD: $\quad$ Parkinson's disease

PolyQ: Polyglutamine

ROS: Radical oxygen species

SCA3: Spinocerebellar ataxia type 3

UPS: Ubiquitin-proteasome system.

\section{Data Availability}

The data used to support the findings of this study are available from the corresponding author upon request.

\section{Disclosure}

I-Cheng Chen's current address is Yushen Orbits Co. Ltd., Taipei City 114, Taiwan.

\section{Conflicts of Interest}

The authors declare that they have no conflicts of interest.

\section{Authors' Contributions}

Chiung-Mei Chen designed the research, revised the paper, and provided financial support; I-Cheng Chen performed the experiments, analyzed the data, and wrote the manuscript; Kuo-Hsuan Chang advised on project design, edited the manuscript, and provided financial support; Yi-Jing Chen established iPSC lines for this study; Yi-Chun Chen and Guey-Jen Lee-Chen commented on the experiment design. All authors approved the final version of the manuscript. I-Cheng Chen and Kuo-Hsuan Chang contributed equally to this study.

\section{Acknowledgments}

The authors would like to thank the study participants. We are also thankful for the technical supports from the Microscope Core Laboratory, Chang Gung Memorial Hospital, Linkou, and Molecular Imaging Core Facility of National Taiwan Normal University. This work was supported by grant MOST-105-2314-B-182A-008-MY2 from the Ministry of Science and Technology, Taipei, and CMRPG3H101, 3G052, and 3A0691-3 from Chang Gung Memorial Hospital, Taoyuan, Taiwan.

\section{Supplementary Materials}

Supplementary Figure 1: effects of NH037 and daidzein on proteasome function and oxidative stress in NC- or SCA3iPSC-derived neurons. (a) Proteasome activity of iPSCderived neurons treated with $1 \mathrm{mg} / \mathrm{ml} \mathrm{NH037} \mathrm{or} 50 \mu \mathrm{M}$ daidzein. The proteasome activity of untreated neurons was normalized as $100 \%$. (b) Lipid peroxidation MDA assay was conducted to evaluate oxidative stress. MDA level of untreated neurons was normalized as $100 \%$. Each experiment for each sample was performed in triplicate. $p$ values: NH037- or daidzein-treated vs. untreated, ${ }^{*} p<0.05$ and ${ }^{* *} p<0.01$. Supplementary Figure 2: the effects of NH037 and daidzein on cytotoxicity and caspase 3 activity in NCor SCA3-iPSC-derived neurons. (a) LDH assay in iPSCderived neurons treated with $1 \mathrm{mg} / \mathrm{ml} \mathrm{NH037} \mathrm{or} 50 \mu \mathrm{M}$ daidzein (untreated neurons was normalized as 100\%). (b) Caspase 3 activity in iPSC-derived neurons treated with $1 \mathrm{mg} / \mathrm{ml} \mathrm{NH037} \mathrm{or} 50 \mu \mathrm{M}$ daidzein. Untreated neurons were normalized as $100 \%$. Each experiment for each sample was performed in triplicate. $p$ values: NH037- or daidzeintreated vs. untreated, ${ }^{* *} p<0.01$. (Supplementary Materials)

\section{References}

[1] G. Haberhausen, M. S. Damian, F. Leweke, and U. Muller, "Spinocerebellar ataxia, type 3 (SCA3) is genetically identical to Machado-Joseph disease (MJD)," Journal of the Neurological Sciences, vol. 132, no. 1, pp. 71-75, 1995.

[2] L. Schols, P. Bauer, T. Schmidt, T. Schulte, and O. Riess, "Autosomal dominant cerebellar ataxias: clinical features, genetics, and pathogenesis," The Lancet Neurology, vol. 3, no. 5, pp. 291-304, 2004.

[3] Y. Kawaguchi, T. Okamoto, M. Taniwaki et al., "CAG expansions in a novel gene for Machado-Joseph disease at chromosome 14q32.1," Nature Genetics, vol. 8, no. 3, pp. 221-228, 1994.

[4] H. L. Paulson, S. S. Das, P. B. Crino et al., "Machado-Joseph disease gene product is a cytoplasmic protein widely expressed in brain," Annals of Neurology, vol. 41, no. 4, pp. 453-462, 1997.

[5] B. Burnett, F. Li, and R. N. Pittman, "The polyglutamine neurodegenerative protein ataxin-3 binds polyubiquitylated proteins and has ubiquitin protease activity," Human Molecular Genetics, vol. 12, no. 23, pp. 3195-3205, 2003.

[6] B. J. Winborn, S. M. Travis, S. V. Todi et al., "The deubiquitinating enzyme ataxin-3, a polyglutamine disease protein, edits Lys63 linkages in mixed linkage ubiquitin chains," The Journal of Biological Chemistry, vol. 283, no. 39, pp. 26436-26443, 2008.

[7] B. O. Evert, J. Araujo, A. M. Vieira-Saecker et al., "Ataxin-3 represses transcription via chromatin binding, interaction with histone deacetylase 3, and histone deacetylation," The Journal of Neuroscience, vol. 26, no. 44, pp. 11474-11486, 2006.

[8] H. L. Paulson, M. K. Perez, Y. Trottier et al., "Intranuclear inclusions of expanded polyglutamine protein in spinocerebellar ataxia type 3," Neuron, vol. 19, no. 2, pp. 333-344, 1997.

[9] C. Pozzi, M. Valtorta, G. Tedeschi et al., "Study of subcellular localization and proteolysis of ataxin-3," Neurobiology of Disease, vol. 30, no. 2, pp. 190-200, 2008.

[10] M. M. Evers, L. J. Toonen, and W. M. van Roon-Mom, "Ataxin-3 protein and RNA toxicity in spinocerebellar ataxia type 3: current insights and emerging therapeutic strategies," Molecular Neurobiology, vol. 49, no. 3, pp. 1513-1531, 2014.

[11] I. Nascimento-Ferreira, T. Santos-Ferreira, L. Sousa-Ferreira et al., "Overexpression of the autophagic beclin-1 protein clears mutant ataxin-3 and alleviates Machado-Joseph disease," Brain, vol. 134, no. 5, pp. 1400-1415, 2011.

[12] F. M. Menzies, J. Huebener, M. Renna, M. Bonin, O. Riess, and D. C. Rubinsztein, "Autophagy induction reduces mutant ataxin-3 levels and toxicity in a mouse model of spinocerebellar ataxia type 3," Brain, vol. 133, no. 1, pp. 93-104, 2010. 
[13] A. Marcelo, F. Brito, S. Carmo-Silva et al., "Cordycepin activates autophagy through AMPK phosphorylation to reduce abnormalities in Machado-Joseph disease models," Human Molecular Genetics, vol. 28, no. 1, pp. 51-63, 2019.

[14] Q. Zheng, T. Huang, L. Zhang et al., "Dysregulation of ubiquitin-proteasome system in neurodegenerative diseases," Frontiers in Aging Neuroscience, vol. 8, p. 303, 2016.

[15] S. Esteves, S. Duarte-Silva, and P. Maciel, "Discovery of therapeutic approaches for polyglutamine diseases: a summary of recent efforts," Medicinal Research Reviews, vol. 37, no. 4, pp. 860-906, 2017.

[16] C. A. Matos, L. P. de Almeida, and C. Nobrega, "MachadoJoseph disease/spinocerebellar ataxia type 3: lessons from disease pathogenesis and clues into therapy," Journal of Neurochemistry, vol. 148, no. 1, pp. 8-28, 2019.

[17] M. Stark and C. Behl, "The Ginkgo biloba extract EGb 761 modulates proteasome activity and polyglutamine protein aggregation," Evidence-based Complementary and Alternative Medicine, vol. 2014, Article ID 940186, 14 pages, 2014.

[18] U. Ramachandran, A. Manavalan, H. Sundaramurthi et al., "Tianma modulates proteins with various neuro-regenerative modalities in differentiated human neuronal SH-SY5Y cells," Neurochemistry International, vol. 60, no. 8, pp. 827-836, 2012.

[19] I. C. Chen, C. N. Chang, W. L. Chen et al., "Targeting ubiquitin proteasome pathway with traditional Chinese medicine for treatment of spinocerebellar ataxia type 3," The American Journal of Chinese Medicine, vol. 47, no. 1, pp. 63-95, 2019.

[20] K. H. Chang, G. J. Lee-Chen, Y. R. Wu et al., "Impairment of proteasome and anti-oxidative pathways in the induced pluripotent stem cell model for sporadic Parkinson's disease," Parkinsonism \& Related Disorders, vol. 24, pp. 81-88, 2016.

[21] L. Q. Qiu, W. S. Lai, D. J. Stumpo, and P. J. Blackshear, "Mouse embryonic fibroblast cell culture and stimulation," Bio-Protocol, vol. 6, no. 13, 2016.

[22] K. H. Chang, I. C. Chen, H. Y. Lin et al., "The aqueous extract of Glycyrrhiza inflata can upregulate unfolded protein response-mediated chaperones to reduce tau misfolding in cell models of Alzheimer's disease," Drug Design, Development and Therapy, vol. 10, pp. 885-896, 2016.

[23] Y. Trottier, Y. Lutz, G. Stevanin et al., "Polyglutamine expansion as a pathological epitope in Huntington's disease and four dominant cerebellar ataxias," Nature, vol. 378, no. 6555, pp. 403-406, 1995.

[24] M. W. Wooten, X. Hu, J. R. Babu et al., "Signaling, polyubiquitination, trafficking, and inclusions: sequestosome $1 / \mathrm{p} 62$ 's role in neurodegenerative disease," Journal of Biomedicine and Biotechnology, vol. 2006, Article ID 62079, 12 pages, 2006.

[25] Y. Chai, S. L. Koppenhafer, S. J. Shoesmith, M. K. Perez, and H. L. Paulson, "Evidence for proteasome involvement in polyglutamine disease: localization to nuclear inclusions in SCA3/MJD and suppression of polyglutamine aggregation in vitro," Human Molecular Genetics, vol. 8, no. 4, pp. 673$682,1999$.

[26] D. H. Lee and A. L. Goldberg, "Proteasome inhibitors: valuable new tools for cell biologists," Trends in Cell Biology, vol. 8, no. 10, pp. 397-403, 1998.

[27] D. Tsikas, "Assessment of lipid peroxidation by measuring malondialdehyde (MDA) and relatives in biological samples: analytical and biological challenges," Analytical Biochemistry, vol. 524, pp. 13-30, 2017.
[28] O. Cooper, H. Seo, S. Andrabi et al., "Pharmacological rescue of mitochondrial deficits in iPSC-derived neural cells from patients with familial Parkinson's disease," Science Translational Medicine, vol. 4, no. 141, article 141ra90, 2012.

[29] Q. Liu, S. Waltz, G. Woodruff et al., "Effect of potent $\gamma$-secretase modulator in human neurons derived from multiple presenilin 1-induced pluripotent stem cell mutant carriers," JAMA Neurology, vol. 71, no. 12, pp. 1481-1489, 2014.

[30] L. M. Watson, M. M. Wong, and E. B. Becker, "Induced pluripotent stem cell technology for modelling and therapy of cerebellar ataxia," Open Biology, vol. 5, no. 7, article 150056, 2015.

[31] Y. M. Yang, S. K. Gupta, K. J. Kim et al., “A small molecule screen in stem-cell-derived motor neurons identifies a kinase inhibitor as a candidate therapeutic for ALS," Cell Stem Cell, vol. 12, no. 6, pp. 713-726, 2013.

[32] M. M. Cobb, A. Ravisankar, G. Skibinski, and S. Finkbeiner, "iPS cells in the study of PD molecular pathogenesis," Cell and Tissue Research, vol. 373, no. 1, pp. 61-77, 2018.

[33] O. S. Lebedeva and M. A. Lagarkova, "Pluripotent stem cells for modelling and cell therapy of Parkinson's disease," Biochemistry, vol. 83, no. 9, pp. 1046-1056, 2018.

[34] H. A. Rowland, N. M. Hooper, and K. A. B. Kellett, "Modelling sporadic Alzheimer's disease using induced pluripotent stem cells," Neurochemical Research, vol. 43, no. 12, pp. 21792198, 2018.

[35] G. Tong, P. Izquierdo, and R. A. Raashid, "Human induced pluripotent stem cells and the modelling of Alzheimer's disease: the human brain outside the dish," The Open Neurology Journal, vol. 11, no. 1, pp. 27-38, 2017.

[36] L. Liu, J. S. Huang, C. Han et al., "Induced pluripotent stem cells in Huntington's disease: disease modeling and the potential for cell-based therapy," Molecular Neurobiology, vol. 53, no. 10, pp. 6698-6708, 2016.

[37] A. Tousley and K. B. Kegel-Gleason, "Induced pluripotent stem cells in Huntington's disease research: progress and opportunity," Journal of Huntington's Disease, vol. 5, no. 2, pp. 99-131, 2016.

[38] F. Codazzi, A. Hu, M. Rai et al., "Friedreich ataxia-induced pluripotent stem cell-derived neurons show a cellular phenotype that is corrected by a benzamide HDAC inhibitor," Human Molecular Genetics, vol. 25, no. 22, pp. 4847-4855, 2016.

[39] J. Du, E. Campau, E. Soragni et al., "Role of mismatch repair enzymes in GAA.TTC triplet-repeat expansion in Friedreich ataxia induced pluripotent stem cells," The Journal of Biological Chemistry, vol. 287, no. 35, pp. 2986129872, 2012.

[40] A. Eigentler, S. Boesch, R. Schneider, G. Dechant, and R. Nat, "Induced pluripotent stem cells from Friedreich ataxia patients fail to upregulate frataxin during in vitro differentiation to peripheral sensory neurons," Stem Cells and Development, vol. 22, no. 24, pp. 3271-3282, 2013.

[41] A. Hick, M. Wattenhofer-Donze, S. Chintawar et al., "Neurons and cardiomyocytes derived from induced pluripotent stem cells as a model for mitochondrial defects in Friedreich's ataxia," Disease Models \& Mechanisms, vol. 6, no. 3, pp. 608621, 2013.

[42] S. Ku, E. Soragni, E. Campau et al., "Friedreich's ataxia induced pluripotent stem cells model intergenerational 
GAA.TTC triplet repeat instability," Cell Stem Cell, vol. 7, no. 5, pp. 631-637, 2010.

[43] J. Liu, P. J. Verma, M. V. Evans-Galea et al., "Generation of induced pluripotent stem cell lines from Friedreich ataxia patients," Stem Cell Reviews, vol. 7, no. 3, pp. 703-713, 2011.

[44] G. Xia, K. Santostefano, T. Hamazaki et al., "Generation of human-induced pluripotent stem cells to model spinocerebellar ataxia type 2 in vitro," Journal of Molecular Neuroscience, vol. 51, no. 2, pp. 237-248, 2013.

[45] S. K. Hansen, T. C. Stummann, H. Borland et al., "Induced pluripotent stem cell - derived neurons for the study of spinocerebellar ataxia type 3," Stem Cell Research, vol. 17, no. 2, pp. 306-317, 2016.

[46] P. Koch, P. Breuer, M. Peitz et al., "Excitation-induced ataxin3 aggregation in neurons from patients with MachadoJoseph disease," Nature, vol. 480, no. 7378, pp. 543-546, 2011.

[47] Z. Ou, M. Luo, X. Niu et al., "Autophagy promoted the degradation of mutant ATXN3 in neurally differentiated spinocerebellar ataxia-3 human induced pluripotent stem cells," BioMed Research International, vol. 2016, Article ID 6701793, 11 pages, 2016.

[48] S. Ouyang, Y. Xie, Z. Xiong et al., “CRISPR/Cas9-targeted deletion of polyglutamine in spinocerebellar ataxia type 3-derived induced pluripotent stem cells," Stem Cells and Development, vol. 27, no. 11, pp. 756-770, 2018.

[49] Y. Ishida, H. Kawakami, H. Kitajima et al., "Vulnerability of purkinje cells generated from spinocerebellar ataxia type 6 patient-derived iPSCs," Cell Reports, vol. 18, no. 4, pp. 10751076, 2017.

[50] Y. Luo, Y. Fan, B. Zhou, Z. Xu, Y. Chen, and X. Sun, "Generation of induced pluripotent stem cells from skin fibroblasts of a patient with olivopontocerebellar atrophy," The Tohoku Journal of Experimental Medicine, vol. 226, no. 2, pp. 151-159, 2012.

[51] M. M. K. Wong, S. D. Hoekstra, J. Vowles et al., "Neurodegeneration in SCA14 is associated with increased PKC $\gamma$ kinase activity, mislocalization and aggregation," Acta Neuropathologica Communications, vol. 6, no. 1, article 99, 2018.

[52] K. Matsuzono, K. Imamura, N. Murakami et al., "Antisense oligonucleotides reduce RNA foci in spinocerebellar ataxia 36 patient iPSCs," Molecular Therapy - Nucleic Acids, vol. 8, pp. 211-219, 2017.

[53] N. Myeku, H. Wang, and M. E. Figueiredo-Pereira, "cAMP stimulates the ubiquitin/proteasome pathway in rat spinal cord neurons," Neuroscience Letters, vol. 527, no. 2, pp. 126-131, 2012.

[54] P. H. Cheng, C. L. Li, Y. F. Chang et al., "miR-196a ameliorates phenotypes of Huntington disease in cell, transgenic mouse, and induced pluripotent stem cell models," The American Journal of Human Genetics, vol. 93, no. 2, pp. 306-312, 2013.

[55] F. L. Chiu, J. T. Lin, C. Y. Chuang et al., "Elucidating the role of the $\mathrm{A} 2 \mathrm{~A}$ adenosine receptor in neurodegeneration using neurons derived from Huntington's disease iPSCs," Human Molecular Genetics, vol. 24, no. 21, pp. 6066-6079, 2015.

[56] A. S. Dickey, V. V. Pineda, T. Tsunemi et al., "PPAR- $\delta$ is repressed in Huntington's disease, is required for normal neuronal function and can be targeted therapeutically," Nature Medicine, vol. 22, no. 1, pp. 37-45, 2016.
[57] H. Y. Hsiao, F. L. Chiu, C. M. Chen et al., "Inhibition of soluble tumor necrosis factor is therapeutic in Huntington's disease," Human Molecular Genetics, vol. 23, no. 16, pp. 4328-4344, 2014.

[58] X. H. Lu, V. B. Mattis, N. Wang et al., "Targeting ATM ameliorates mutant huntingtin toxicity in cell and animal models of Huntington's disease," Science Translational Medicine, vol. 6, no. 268, article 268ra178, 2014.

[59] V. B. Mattis, C. Tom, S. Akimov et al., "HD iPSC-derived neural progenitors accumulate in culture and are susceptible to BDNF withdrawal due to glutamate toxicity," Human Molecular Genetics, vol. 24, no. 11, pp. 3257-3271, 2015.

[60] W. X. Ding, H. M. Ni, W. Gao et al., "Linking of autophagy to ubiquitin-proteasome system is important for the regulation of endoplasmic reticulum stress and cell viability," The American Journal of Pathology, vol. 171, no. 2, pp. 513-524, 2007.

[61] R. Xiong, D. Siegel, and D. Ross, "The activation sequence of cellular protein handling systems after proteasomal inhibition in dopaminergic cells," Chemico-Biological Interactions, vol. 204, no. 2, pp. 116-124, 2013.

[62] Z. Zhang, T. N. Lam, and Z. Zuo, "Radix Puerariae: an overview of its chemistry, pharmacology, pharmacokinetics, and clinical use," Journal of Clinical Pharmacology, vol. 53, no. 8, pp. 787-811, 2013.

[63] D. M. Tham, C. D. Gardner, and W. L. Haskell, "Potential Health Benefits of Dietary Phytoestrogens: A Review of the Clinical, Epidemiological, and Mechanistic Evidence," The Journal of Clinical Endocrinology and Metabolism, vol. 83, no. 7, pp. 2223-2235, 1998.

[64] H. W. Jung, A. N. Kang, S. Y. Kang, Y. K. Park, and M. Y. Song, "The root extract of Pueraria lobata and its main compound, puerarin, prevent obesity by increasing the energy metabolism in skeletal muscle," Nutrients, vol. 9, no. 1, p. 33, 2017.

[65] D. W. Lim, C. Lee, I. H. Kim, and Y. T. Kim, “Anti-inflammatory effects of total isoflavones from Pueraria lobata on cerebral ischemia in rats," Molecules, vol. 18, no. 9, pp. 1040410412, 2013.

[66] K. B. Magalingam, A. K. Radhakrishnan, and N. Haleagrahara, "Protective mechanisms of flavonoids in Parkinson's disease," Oxidative Medicine and Cellular Longevity, vol. 2015, Article ID 314560, 14 pages, 2015.

[67] P. B. Kaufman, J. A. Duke, H. Brielmann, J. Boik, and J. E. Hoyt, "A comparative survey of leguminous plants as sources of the isoflavones, genistein and daidzein: implications for human nutrition and health," Journal of Alternative and Complementary Medicine, vol. 3, no. 1, pp. 7-12, 1997.

[68] A. Kirakosyan, P. B. Kaufman, S. Warber, S. Bolling, S. C. Chang, and J. A. Duke, "Quantification of major isoflavonoids and L-canavanine in several organs of kudzu vine (Pueraria montana) and in starch samples derived from kudzu roots," Plant Science, vol. 164, no. 5, pp. 883-888, 2003.

[69] R. C. Choi, J. T. Zhu, A. W. Yung et al., "Synergistic action of flavonoids, baicalein, and daidzein in estrogenic and neuroprotective effects: a development of potential health products and therapeutic drugs against Alzheimer's disease," Evidence-based Complementary and Alternative Medicine, vol. 2013, Article ID 635694, 10 pages, 2013.

[70] M. Pan, H. Han, C. Zhong, and Q. Geng, "Effects of genistein and daidzein on hippocampus neuronal cell proliferation and BDNF expression in H19-7 neural cell line," The Journal of Nutrition, Health \& Aging, vol. 16, no. 4, pp. 389-394, 2012. 
[71] S. H. Yang, C. C. Liao, Y. Chen, J. P. Syu, C. J. Jeng, and S. M. Wang, "Daidzein induces neuritogenesis in DRG neuronal cultures," Journal of Biomedical Science, vol. 19, no. 1, p. 80, 2012.

[72] L. M. Watson, M. M. K. Wong, J. Vowles, S. A. Cowley, and E. B. E. Becker, "A simplified method for generating Purkinje cells from human-induced pluripotent stem cells," Cerebellum, vol. 17, no. 4, pp. 419-427, 2018. 


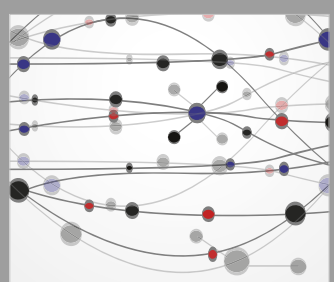

The Scientific World Journal
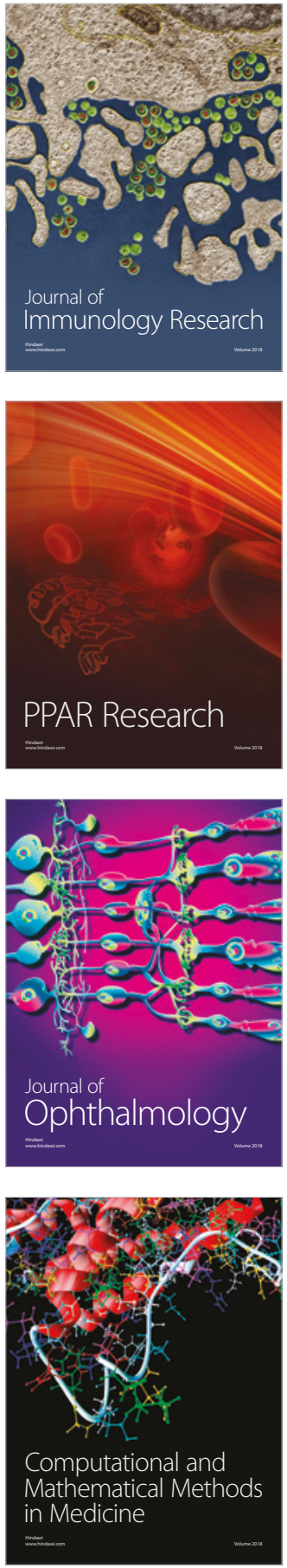

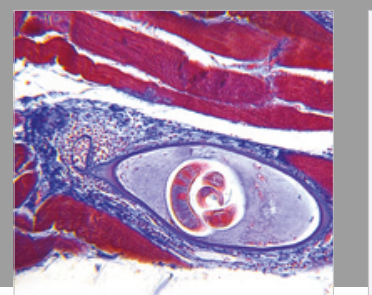

Gastroenterology Research and Practice

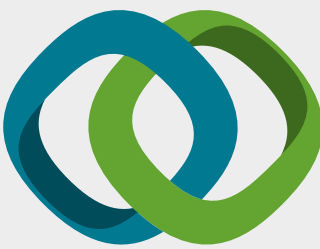

\section{Hindawi}

Submit your manuscripts at

www.hindawi.com
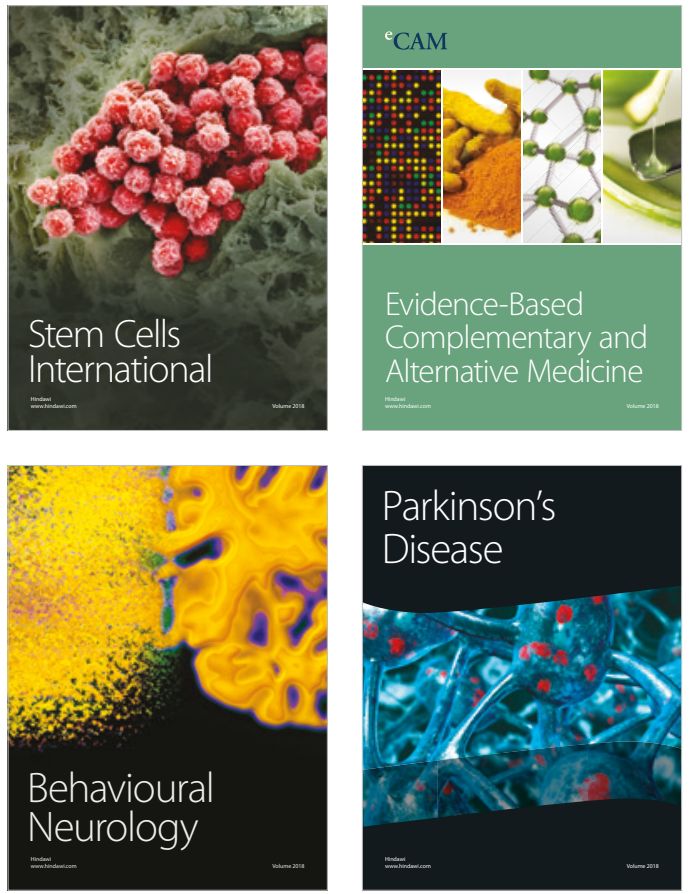

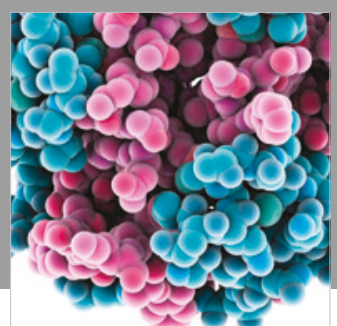

ournal of

Diabetes Research

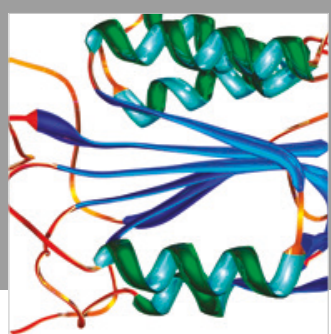

Disease Markers
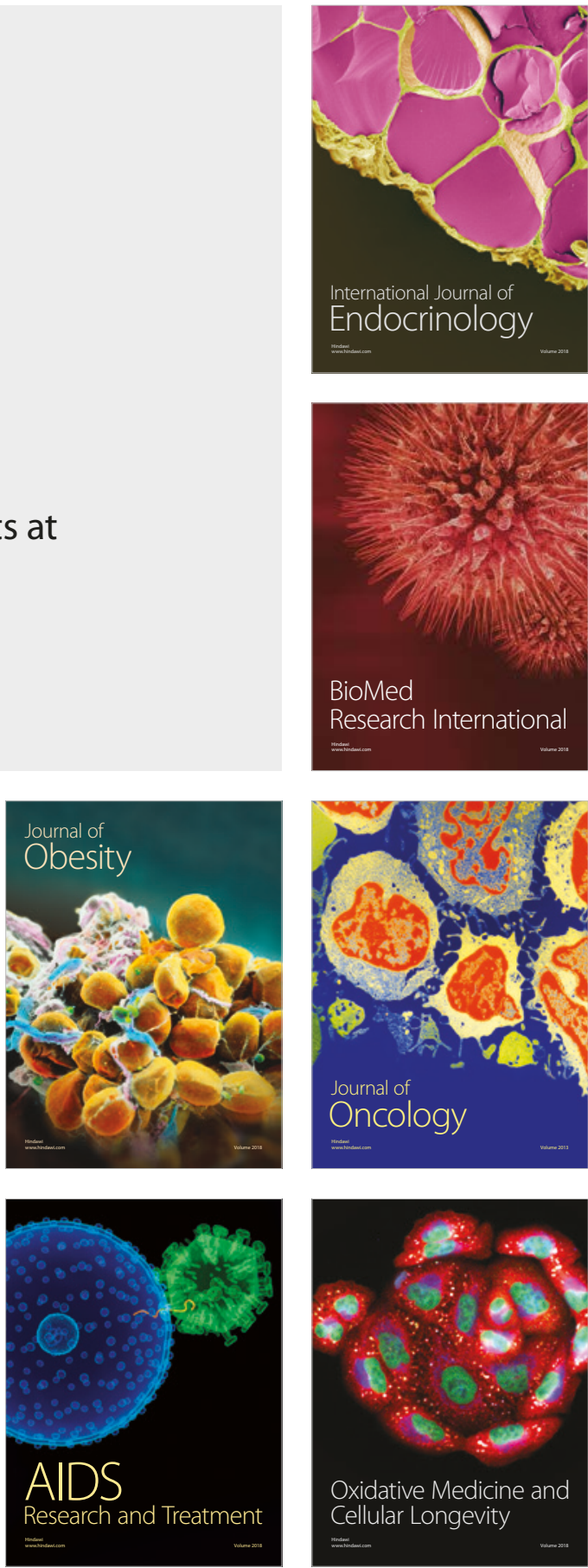